\title{
IS IT ALWAYS DARK IN CAVES?
}

\author{
Giovanni BADINO \\ Dipartimento di Fisica Generale, Università di Torino \\ Associazione Culturale La Venta
}

\begin{abstract}
Underground natural sources of visible light are considered. The main light producer is Cerenkov radiation emitted in air, water and rock by cosmic ray muons, that depends, in a complex way, on shape of mountain and of caves. In general the illumination increases linearly with the cavity dimensions. Other light sources are from secondary processes generated by radioactive decays in rock from minerals luminescence. The natural light fluxes in caves are in general easy to detect but are not used from underground life.
\end{abstract}

\section{RIASSUNTO (È sempre buio in grotta ?)}

Vengono qui analizzate le sorgenti naturali di luce visibile sottoterra. La maggiore fonte ne è la radiazione Cerenkov emessa in aria, acqua e roccia dai muoni della radiazione cosmica che dipende, in modo complesso, dalle forme della cavità e della montagna; in generale essa cresce linearmente con le dimensioni dell' ambiente. Altra luce viene prodotta da processi secondari generati dai decadimenti radioattivi nella roccia e dalla luminescenza dei minerali. I flussi di luce visibile sottoterra sono in linea di massima facili da misurare ma non vengono utilizzati dalle forme di vita sotterranea.

Keywords: muons underground - natural light in caves - Cerenkov radiation - caves darkness Vostok lake - caves energy balance - rock radioactivity - beta decays - radon in caves

\section{Introduction}

It is widely known that caves are dark. This darkness stimulates the people's curiosity: "is it always dark in caves?" is a very usual question asked to cavers. So common that the author himself asked it to the caver that was introducing him to speleology. This memory, together with his work in underground neutrino astronomy, has pushed him to give a more detailed answer to the question. It is always dark in caves, yes: but not so absolutely dark...

We are going to consider an extended range of visible light. It would be necessary to distinguish between photopic and scotopic conditions of sight, but we may say that under ordinary daylight conditions the relative luminous efficiency of human eyes is maximal at a wavelength of $560 \mathrm{~nm}$, which corresponds to the yellow-green region. 
From this maximum visibility falls asymptotically toward both ends of the spectrum: the maximum is $10^{-3}$ at wavelengths of $410 \mathrm{~nm}$ (violet) and $720 \mathrm{~nm}$ (red) (Thewlis, 1962). But we shall keep the range $350-800 \mathrm{~nm}$ to cover different eyes.

Traces of visible light are produced even very deep underground (or underwater) by cosmic rays and radioactive decays, so much that many experiments use this light to study radiation (Cecchini, 2001).

We are going to see how light and particles are connected.

In this work we use only mechanic units (W) and not the usual illumination units (lumen and lux) for simplicity. We remember that a lumen is equal to $1 / 680 \mathrm{~W}$ emitted at $560 \mathrm{~nm}$ by a monochromatic source ( $1 \mathrm{~W}$ of radiant energy like this is sometimes called "lightwatt"). Likewise, a lux (lumen $\mathrm{m}^{-2}$ ) is equivalent to $1 / 680 \mathrm{Wm}^{-2}$.

\section{The black body radiation}

First of all, let us consider the black body radiation, that is, the radiation in thermal equilibrium with matter at an absolute temperature $\mathrm{T}$.

A good example of this type of radiation comes from the Sun, which radiates almost exactly as a black body at a temperature of $5800 \mathrm{~K}$. The relation between the wavelength of maximum power release and the temperature $\mathrm{T}$ is the Wien displacement law (Richtmyer \& Kennard, 1955):

$$
\lambda_{m} T=0.003 \mathrm{~K} \mathrm{~m}
$$

For instance, we may calculate the wavelength of the Sun maximum power: $520 \mathrm{~nm}$. Actually the Sun is mainly yellow.

Also black bodies at room temperature radiate, but the wavelength of maximum power release is around $10 \mathrm{~mm}$, that corresponds to far infrared.

Anyway the radiation is not only at $\lambda_{\mathrm{m}}$ : the emission covers a wide wavelength range around it. Planck's Law for the black body radiant energy per surface unit in the wavelength range $\mathrm{d} \lambda$ gives the spectrum:

$$
\varepsilon_{b b}(\lambda) d \lambda=\frac{2 \pi h c^{2}}{\lambda^{5}} \frac{1}{\exp \left(\frac{h c}{k \lambda T}\right)-1} d \lambda
$$

Where $\mathrm{c}=3 \times 10^{8}$ (light velocity), $\mathrm{k}=1.4 \times 10^{-23}$ (Boltzmann constant), $\mathrm{h}=6.6 \times 10^{-34}$ (Planck constant) in SI units. The equation shows that there is an extremely quick $\left(-\lambda^{-5} \exp (-\operatorname{const} / \lambda)-\right)$ decrease for wavelengths shorter than the maximum. This also allows us to calculate the visible radiation flux coming from a black body source at caves temperature.

Here we are interested in temperatures ranging from $\mathrm{T}=270 \mathrm{~K}$ up to $\mathrm{T}=320 \mathrm{~K}$. 
Table 1 - Black body spectrum from $270 \mathrm{~K}$ to $320 \mathrm{~K}$ in phtons $\mathrm{m}^{-2} \mathrm{~s}^{-1}$

\begin{tabular}{|c|c|c|c|}
\hline Temperature & $270 \mathrm{~K}\left(-3^{\circ} \mathrm{C}\right)$ & $295 \mathrm{~K}\left(22^{\circ} \mathrm{C}\right)$ & $320 \mathrm{~K}\left(47^{\circ} \mathrm{C}\right)$ \\
\hline Maximum emission $\lambda_{\mathrm{m}} \mathrm{nm}$ & $11100 \mathrm{~nm}$ & $10200 \mathrm{~nm}$ & $9400 \mathrm{~nm}$ \\
\hline $350-400$ & 0 & 0 & 0 \\
\hline $400-450$ & 0 & 0 & 0 \\
\hline $450-500$ & 0 & 0 & 0 \\
\hline $500-550$ & 0 & 0 & $1 \times 10^{-10}$ \\
\hline $550-600$ & 0 & $2 \times 10^{-10}$ & $2 \times 10^{-7}$ \\
\hline $600-650$ & $10^{-10}$ & $1 \times 10^{-7}$ & $7 \times 10^{-5}$ \\
\hline $650-700$ & $4 \times 10^{-8}$ & $4 \times 10^{-5}$ & $1 \times 10^{-2}$ \\
\hline $700-750$ & $8 \times 10^{-6}$ & $4 \times 10^{-3}$ & 0.7 \\
\hline $750-800$ & $7 \times 10^{-4}$ & 0.2 & 31 \\
\hline
\end{tabular}

We may see (!) that also in the case of really hot caves $\left(47^{\circ} \mathrm{C}\right)$ it is necessary to wait minutes to detect a single visible, deep red photon $(650 \mathrm{~nm})$ from a square meter of wall (or air).

Let us nevertheless note that, also in the case of an high emission, the black body radiation cannot be used for vision, a process that requires a contrast among objects: the black body radiation is a photon gas in maximum entropy state, it fills all the space and is emitted by every part of the system, including the eyes (or the film). It can be used for sight only if the system and the source are completely separated and not in thermal equilibrium, as it happens with our eyes and the Sun or light bulb filaments.

\section{The Cerenkov radiation}

Underground cavities, even at large depth, are crossed by charged particle radiation coming from outer space (mainly muons and neutrinos from cosmic rays) and locally produced by radioactive decays (mainly electrons from beta-decays). This radiation can produce detectable photons fluxes by Cerenkov emission.

Let us see some details on this mechanism.

It is well known that particles of real, non-zero mass, cannot attain the speed of light in vacuum, $c=3 \times 10^{8} \mathrm{~m} / \mathrm{s}$. But in dielectric materials like crystals, water or air the light has a phase speed lower than light, and the particles may go faster than photons. In this case the interaction between matter's dipoles and the particle's travelling field produce a light release, the Cerenkov light, named from the Russian physicist who first studied this process in the 1930 (Jelley, 1959). 
This light cannot overtake the particle: analogously to a supersonic plane travelling in the vertex of the cone of its shock wave, the particle flies in the vertex of a photons cone emitted by itself.

Let us call " $n$ " the refractive index of a material. In this case the phase velocity c' of light inside it is:

$$
c^{\prime}=\frac{c}{n}
$$

And the angle between the trajectory of the particle travelling at speed $\mathrm{v}$ and the direction of emitted photons is given by:

$$
\theta_{c}=\arccos \left(\frac{c}{v n}\right)
$$

That has a solution only if the particle travels faster than light in the material

$$
v \geq \frac{c}{n}=c^{\prime}
$$

This speed corresponds to the particle's minimum total $\mathrm{E}_{\mathrm{th}}$ and kinetic $\mathrm{K}_{\mathrm{th}}$ energy given by:

$$
\begin{aligned}
E_{t h} & =\frac{m_{0} c^{2}}{\sqrt{1-\left(\frac{v}{c}\right)^{2}}}=\frac{m_{0} c^{2}}{\sqrt{1-\left(\frac{1}{n}\right)^{2}}} \\
K_{t h} & =E_{t h}-m_{0} c^{2}
\end{aligned}
$$

The photons wavelength (its colour) depends upon the dielectric detailed physical properties; but it is possible to demonstrate that generally the photons are emitted in the frequency range of blue and nearest UV. We are going to consider the wavelength between 350 and $500 \mathrm{~nm}$, the most used range for Cerenkov light practical uses (Jelley, 1959).

The number of emitted photons per meter of flight in the wavelength range between $\lambda_{1}$ and $\lambda_{2}$ is given by:

$$
\frac{d N}{d l}=2 \pi \alpha\left(1-\frac{c^{2}}{v^{2} n^{2}}\right)\left(\frac{1}{\lambda_{2}}-\frac{1}{\lambda_{1}}\right) \approx 0.0459\left(1-\frac{c^{2}}{v^{2} n^{2}}\right)\left(\frac{1}{\lambda_{2}}-\frac{1}{\lambda_{1}}\right)
$$

Where $\alpha=1 / 137$ is the fine-structure constant; this shows that the power emission per 
emission increases strongly as 1 decreases. The total emitted energy does not diverges because also the refractive index $\mathrm{n}$ depends upon 1 and the radiation release is limited to the $n>1$ region that extends up to the near UV.

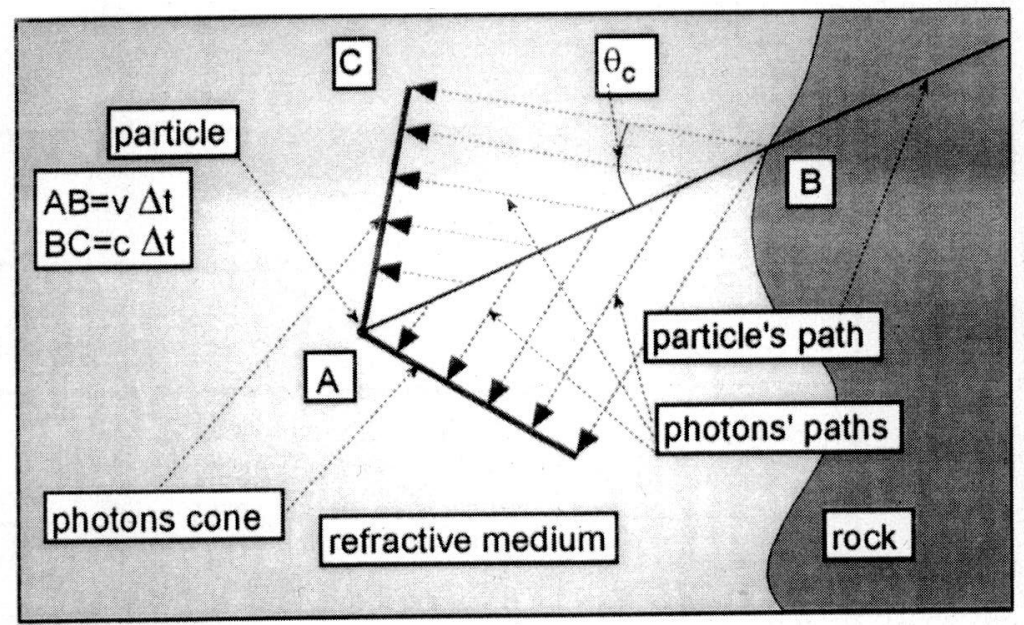

Fig. 1 - The general shape of Cerenkov radiation in air (or water) emitted by a particle coming from the rock: the photons are arranged on a conic surface with vertex on the particle.

Therefore the emission rate depends upon the velocity of the particle, i.e. on his energy. If we consider the relativistic kinetic energy of a particle $m_{0}$ :

$$
K=m_{0} c^{2}\left(\frac{1}{\sqrt{1-\left(\frac{v}{c}\right)^{2}}}-1\right)
$$

We may re-write the Cerenkov emission rate as

$$
\frac{d N}{d l}=0.0459\left(\frac{1}{\lambda_{2}}-\frac{1}{\lambda_{1}}\right)\left\{1-\frac{1}{n^{2}}\left[1-\left(\frac{m_{0} c^{2}}{K+m_{0} c^{2}}\right)^{2}\right]^{-1}\right\}
$$

It is easy to see that the emission starts at a minimum kinetic energy $\mathrm{K}_{\mathrm{th}}$, as we have already discussed, and increases very quickly up to an asymptotic value when the particle speed approaches c: 


$$
\left(\frac{d N}{d l}\right)_{\infty}=0.0459\left(\frac{1}{\lambda_{2}}-\frac{1}{\lambda_{1}}\right)\left(1-\frac{1}{n^{2}}\right)=\Gamma_{\infty}
$$

The graphic shows an example: the emission rate of an electron travelling in water as function of its kinetic energy.

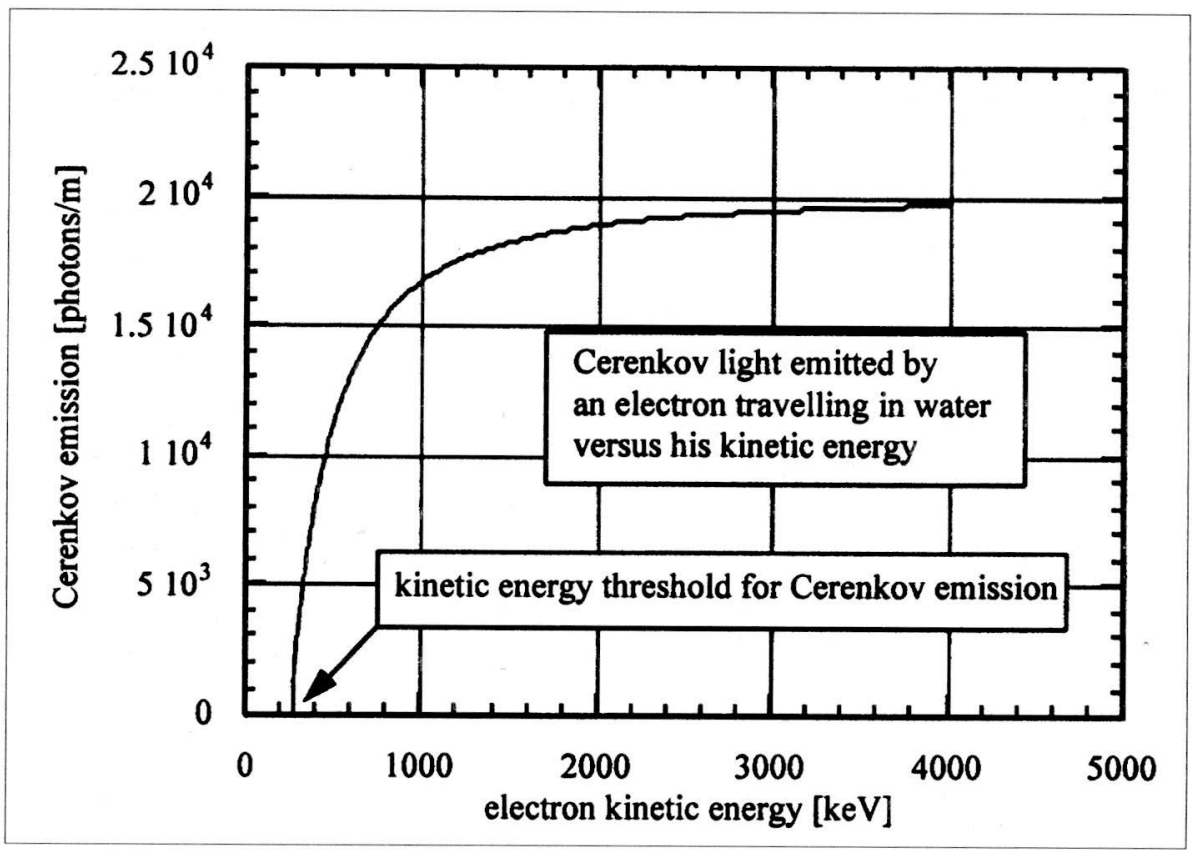

Fig. 2 - Emission rate of an electron travelling in water as function of its kinetic energy.

It is important to note that, in this case, the emission rate reaches $90 \%$ of asymptotic value $\Gamma_{\infty}$ at $\mathrm{K}=1400 \mathrm{keV}$.

This means that we may regard the emission rate (and the emission angle) as constant above kinetic energies 10 times larger than the threshold.

For this reason, at very high kinetic energies (like those of cosmic rays), the light emission is constant and independent from energy; anyhow, we are also going to consider particles emitting at "low" energies when studying the beta decays contribution to caves illumination.

Table 2 resumes these results for caves interesting dielectric materials: air, water and calcite. 
Table 2 - Cerenkov emission parameters for materials responsible of underground light releases.

\begin{tabular}{|c|c|c|c|c|}
\hline Material & Air & Water & $\begin{array}{c}\text { Calcite } \\
\text { (ord. ray) }\end{array}$ & $\begin{array}{c}\text { Calcite } \\
\text { (extr. ray) }\end{array}$ \\
\hline $\mathrm{n}$ : refractive index (at $\lambda=434 \mathrm{~nm}$ ) & 1.00029 & 1.33 & 1.68 & 1.49 \\
\hline $\begin{array}{l}\mathrm{K}_{\mathrm{th}}: \text { electrons kinetic energy threshold for } \\
\text { Cerenkov emission }[\mathrm{MeV}] \\
\mathrm{m}_{0} \mathrm{c}^{2}=0.511 \mathrm{MeV}\end{array}$ & 20.7 & 0.264 & 0.125 & 0.178 \\
\hline $\begin{array}{c}\mathrm{K}_{\mathrm{th}}: \text { muons kinetic energy threshold for } \\
\text { Cerenkov emission }[\mathrm{MeV}] \\
\mathrm{m}_{0} \mathrm{c}^{2}=105.7 \mathrm{MeV} \\
\end{array}$ & 4280 & 54.6 & 25.8 & 36.9 \\
\hline kinetic energy at $90 \%$ of emission & $130 \mathrm{~m}_{0} \mathrm{c}^{2}$ & $2.74 \mathrm{~m}_{0} \mathrm{c}^{2}$ & $1.55 \mathrm{~m}_{0} \mathrm{c}^{2}$ & $2.03 \mathrm{~m}_{0} \mathrm{c}^{2}$ \\
\hline$\theta_{\mathrm{c} \infty}:$ emission angle at $\mathrm{v} \approx \mathrm{c}$ & $1.4^{\circ}$ & $41^{\circ}$ & $53^{\circ}$ & $48^{\circ}$ \\
\hline $\begin{array}{c}\Gamma_{\infty}: \text { number of photons at } \mathrm{v} \approx \mathrm{c}[\text { photons } / \mathrm{m}] \\
(350-500 \mathrm{~nm})\end{array}$ & 26 & 20000 & 30000 & 25000 \\
\hline
\end{tabular}

The refractive index of air linearly depends on pressure (here the sea-level value is given), but we may consider it as constant in the caves altitude range up to 2-3000 $\mathrm{m}$ asl. The electron kinetic energy threshold for Cerenkov emission is $20 \mathrm{MeV}$, an order of magnitude larger than the typical energies of radioactive sources, $1 \mathrm{MeV}$; we can be sure that there is no air Cerenkov emission due to beta decays. The muon's minimum energy for light emission in air is $4 \mathrm{GeV}$, very typical of cosmic rays muons underground: we are going to see that this is the main light source in caves.

The energy at $90 \%$ of emission gives us the energy level above which we may consider that the particle emits at constant rate, which is $v^{\mathrm{a}} \mathrm{c}$ for Cerenkov emission.

We also see that the Cerenkov emission angle in air $($ at $v \approx c)$ is very small, around $1.5^{\circ}$, which means that the photons travel in a "plate" around the particle. In water and calcite the light is instead emitted at high angles from the particle line of flight and the illumination is stronger and more isotropic.

\section{The cosmic rays}

We must consider some more details about the radiation reaching underground with us.

The interstellar space is filled with an extremely tenuous high-energy radiation (the so called "primary cosmic rays") essentially composed by nuclei (mainly proton and alpha particles) with a power law spectrum energy ranging from $10^{10} \mathrm{eV}$ up to $10^{20}$ $\mathrm{eV}$ (Gaisser, 1990). This radiation cannot reach the ground because it is extremely nuclear reactive and interacts with air nuclei at altitudes around 15-20 km above sea level, producing showers of particles of all kinds ("Extensive Air Showers"), the so 
called "secondary cosmic rays". At sea level, electrons, gammas, neutrinos and muons compose this radiation for the most part.

We may consider this flux as isotropic, to say, its intensity does not depend on the direction: the sky appears almost uniformly "lighted" by this radiation.

Table 3 gives some typical values of charged particles fluxes at sea level (Particle Data Group, 1986).

Table 3 - Some typical values of charged particles fluxes at sea level (Particle Data Group, 1986).

\begin{tabular}{|l|c|c|}
\hline Cosmic ray component & Muons & Electrons and others \\
\hline Total flux crossing unit horizontal area from above $\left[\mathrm{m}^{-2} \mathrm{~s}^{-1}\right]$ & 130 & 50 \\
\hline $\begin{array}{l}\text { Total flux from above, impinging on a sphere of unit cross- } \\
\text { sectional area }\left[\mathrm{m}^{-2} \mathrm{~s}^{-1}\right]\end{array}$ & 170 & 70 \\
\hline
\end{tabular}

Note the difference between the second and third rows: the second is referred to a flux on a flat surface, which for high incidence angles appears very small. The third "shows", in any case, "one square meter" to the incoming particles.

The electrons and other charged particles, and the gamma-photons, are called "soft component" because they are completely stopped by ground surface. Muons and neutrinos are referred to as "hard component" because they continue their flight underground.

So just few meters below the surface only muons and neutrinos are present. We are going to consider the neutrinos role, important only at rock depths exceeding 1-2 km, only at the end of this work.

\section{The muons interaction in rock}

Table 4 shows the electron and muon main physical parameters. The muon is a fundamental particle (point-like, basing upon actual knowledge) very similar to the electron, of which it may be considered an excited state. The only difference is the mass (muon mass is 206.8 times larger than the electron mass) and its instability: its mean life is $2.2 \mu$ s and it decays into an electron and two neutrinos (Segre, 1965).

Table 4 - Electron and muon main physical parameters

\begin{tabular}{|c|c|c|}
\hline Particle & $\mathrm{m}_{\mathrm{c}} \mathrm{c}^{2}$ & Mean life \\
\hline Electron & $0.511 \mathrm{MeV}$ & Stable \\
\hline Muon & $105.7 \mathrm{MeV}$ & $2.2 \mu \mathrm{s}$ \\
\hline
\end{tabular}


We are going to consider the contribution to caves Cerenkov lighting due to high energy cosmic rays muons in air, water and rock and to low energy electrons from beta decay in rock.

The muons interact with matter and lose energy at a quite constant rate: in limestone (or dolomite) they lose around $0.7 \mathrm{Gev}$ per meter of flight. This means that, very roughly, a muon entering underground with energy of $20 \mathrm{GeV}$ will have $13 \mathrm{Gev}$ after ten meters of crossed rock, $6 \mathrm{GeV}$ after 20 meters and it will reach 30 meters of depth.

We may generalise and calculate the minimum energy $E_{\min }$ to reach a depth $x$, that is, to arrive there with zero energy.

If we include all the processes of interaction muon-rock we have (Hayakawa, 1969):

$$
\begin{aligned}
& E_{\min }=\frac{a}{b}\left[\exp \left(b \rho_{r} x\right)-1\right] \quad \mathrm{GeV} \mathrm{m}^{-1} \text { of limestone } \\
& a=2.5 \times 10^{-4} \mathrm{GeV} \mathrm{m}^{2} \mathrm{~kg}^{-1} \\
& b=4.1 \times 10^{-7} \mathrm{~m}^{2} \mathrm{~kg}^{-1} \\
& \rho_{r}=2700 \mathrm{~kg} \mathrm{~m}^{-3}
\end{aligned}
$$

Where "a" accounts for ionisation losses, " $b$ " for bremsstrahlung losses and " $\rho_{r}$ " is a typical limestone density. The graphic shows the result:

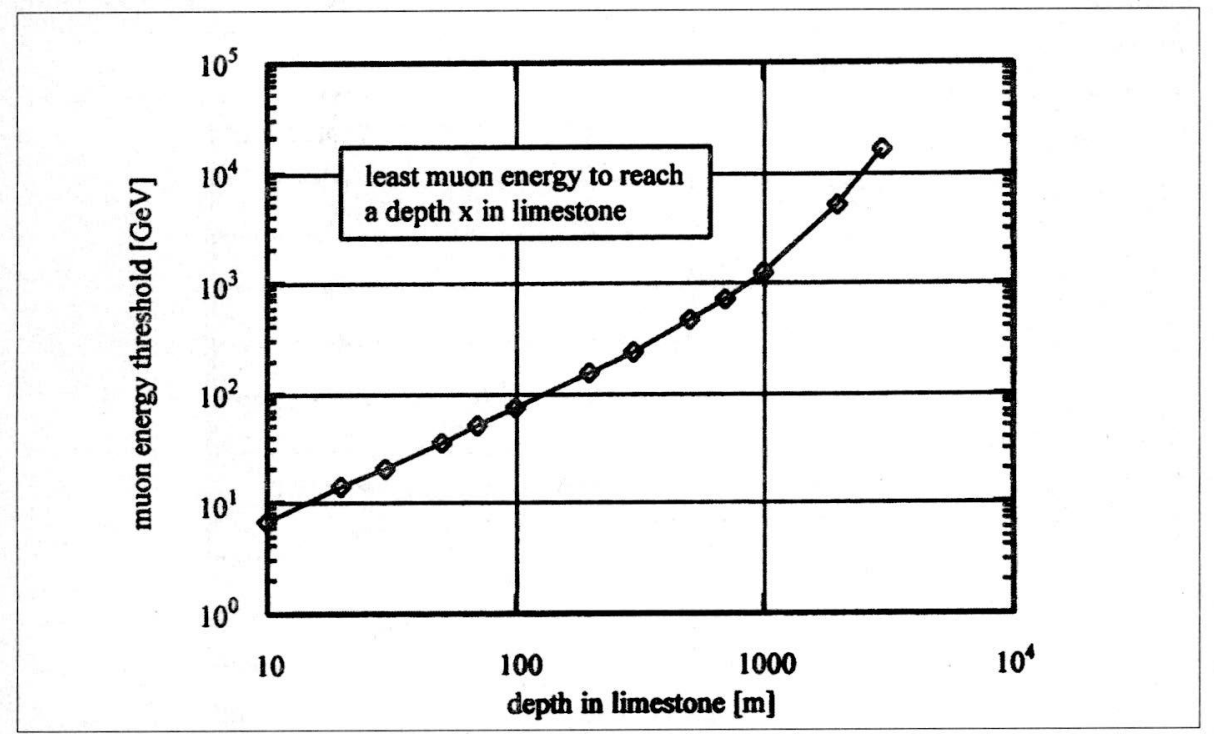

Fig. 3 - Least muon energy to reach a depth $x$ in limestone 
The formula may be simplified if the bremsstrahlung processes are negligible, i.e. when the argument of exponential is very small. In this case, correct for $z<<1000$ meters, we have:

$$
E_{\min } \approx \frac{a}{b}\left(b \rho_{r} z\right)=0.67 z \mathrm{GeV} \mathrm{m}^{-1} \text { of limestone }
$$

That corresponds to the linear first part of the graphic. It is possible to see that the muon energies necessary to reach the actually known typical caves $(10<x<500 \mathrm{~m})$ range from 10 to $700 \mathrm{GeV}$.

\section{The muons energy and intensity}

We have already noted that the energy spectrum of primary cosmic rays in outer space has power-law behaviour. The muons too behave in this way: at the surface the flux of vertical muons with energy greater than $\mathrm{E}$ (in $\mathrm{GeV}$ ) is given by:

$$
I_{\text {ver, }, \mu}(>E) \approx 1450 E^{-1.78}\left(\frac{115}{115+1.1 E}\right) \mathrm{m}^{-2} \mathrm{~s}^{-1} \mathrm{sr}^{-1}
$$

A formula useful for $10<\mathrm{E}<1000 \mathrm{GeV}$ that we have obtained simplifying the data given in (Ambrosio et al., 1995) to fit our energy range.

This shows, for instance, that increasing the energy threshold (or the depth) of a factor 10 the flux is reduced by a factor 60 .

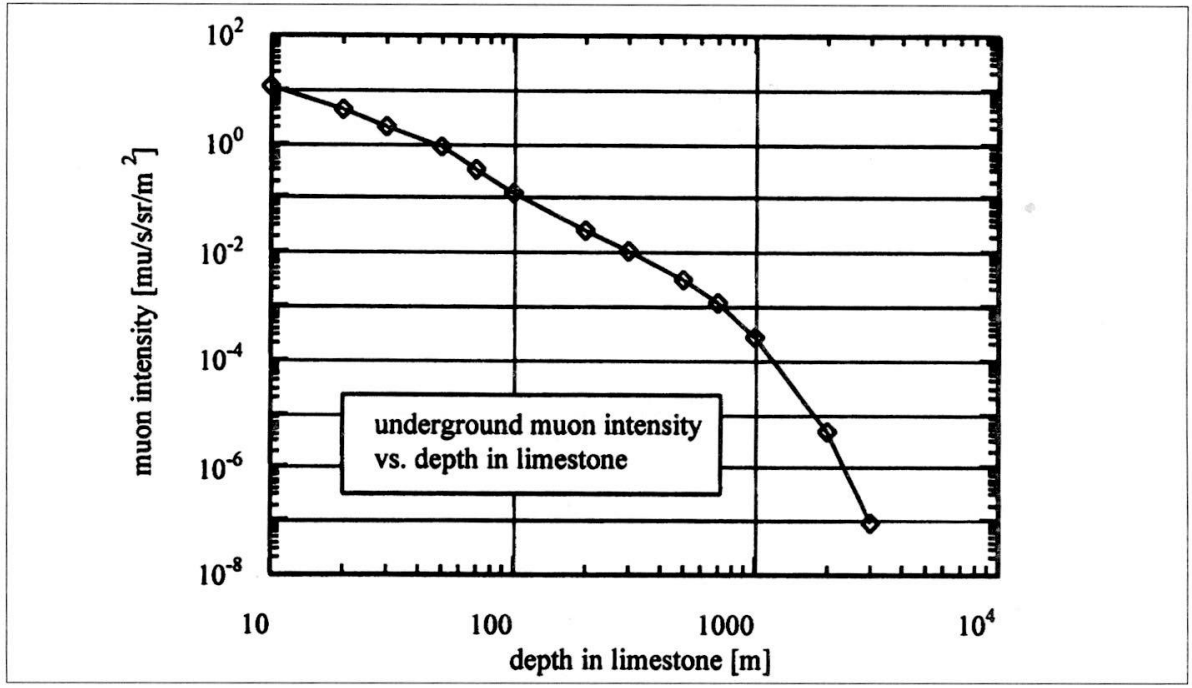

Fig. 4 - Muons intensity versus depth in limestone. 
The graphic shows the result, that is the number of muons versus depth in limestone (Hayakawa, 1969). Note that the parameter is the muon intensity, that is, the number of particles per second per square meter per steradian.

We may now solve our first question: how many muons cross a surface "S" in a cave? We assume a polar coordinate system, centred on $\mathrm{S}$.

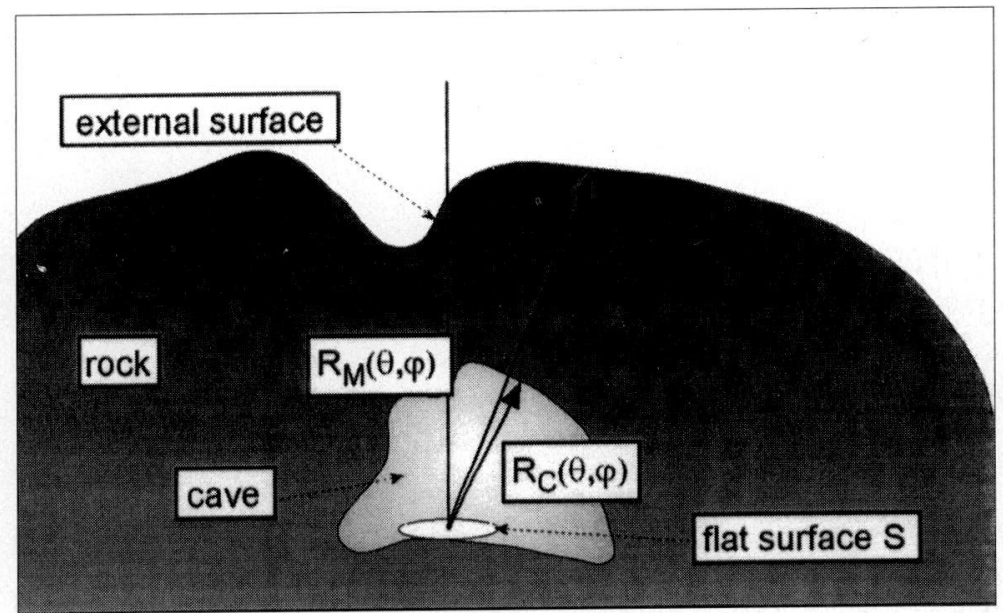

Fig. 5 - The main parameters for Cerenkov emission undergound are two thickness in each direction: that of rock, responsible of muons filtering, and that of air, responsible of light emission.

Let us call $\mathrm{R}_{\mathrm{M}}(\theta, \varphi)$ the function that gives us the position of ground surface in the direction $(\theta, \varphi)$, and $\mathrm{R}_{C}(\theta, \varphi)$ the position of cave wall surfaces in the same direction. The thickness of rock is then obviously given by the difference $R_{M}(\theta, \varphi)-R_{C}(\theta, \varphi)$. Let us consider now a solid angle $\mathrm{d} \Omega$ in the direction $(\theta, \varphi)$. The total flux crossing our surface is given by:

$$
\begin{aligned}
& F(\theta, \varphi)=I_{\mu}\left(R_{\text {rock }}(\theta, \varphi)\right) S \cos \theta d \Omega \\
& R_{\text {rock }}(\theta, \varphi)=R_{M}(\theta, \varphi)-R_{C}(\theta, \varphi)
\end{aligned}
$$

The functions $\mathrm{R}_{\mathrm{M}}(\theta, \varphi)$ are in general very complex and only in special cases, like for large underground cosmic ray observatories, they are calculated. For this reason, to estimate these fluxes we have to make some radical approximations.

The first one is to suppose that the muon flux at the surface is independent from q: we may then use the vertical intensity in all directions, that is: 


$$
I_{\mu}\left(R_{M}(\theta, \varphi)\right) \approx I_{v e r, \mu}\left(>E_{\min } R_{M}(0, \varphi)\right)
$$

The second assumption is that the $\mathrm{R}_{\mathrm{M}}(\theta, \varphi)$ behaves in such a way allowing us to consider only the contribution of muons coming from the direction $\left(\theta_{0}, \varphi_{0}\right)$ and around it, where $R_{M}$ is minimum:

$$
\begin{aligned}
& \theta_{0}, \varphi_{0} \leftrightarrow R_{M}\left(\theta_{0}, \varphi_{0}\right)=\min \left[R_{M}(\theta, \varphi)\right] \\
& x_{0} \approx R_{M}\left(\theta_{0}, \varphi_{0}\right)
\end{aligned}
$$

The third approximation is to assume regularity and "flatness" for the mountain surface around the direction $\left(\theta_{0}, \varphi_{0}\right)$ : this is the case, for example, of a cave below a plateau. We then exclude a very complex surface structure around the cave.

We may therefore assume that the muon intensity around $\left(\theta_{0}, \varphi_{0}\right)$ behaves like:

$$
I_{\mu}(\theta, \varphi) \approx I_{\mu}\left(x_{0}\right) \cos ^{n} \theta_{0}
$$

The index " $\mathrm{n}$ " is a function of depth and ranges from 1.5 at 10 meters up to 3.5 at 1000; the next graphic shows this function:

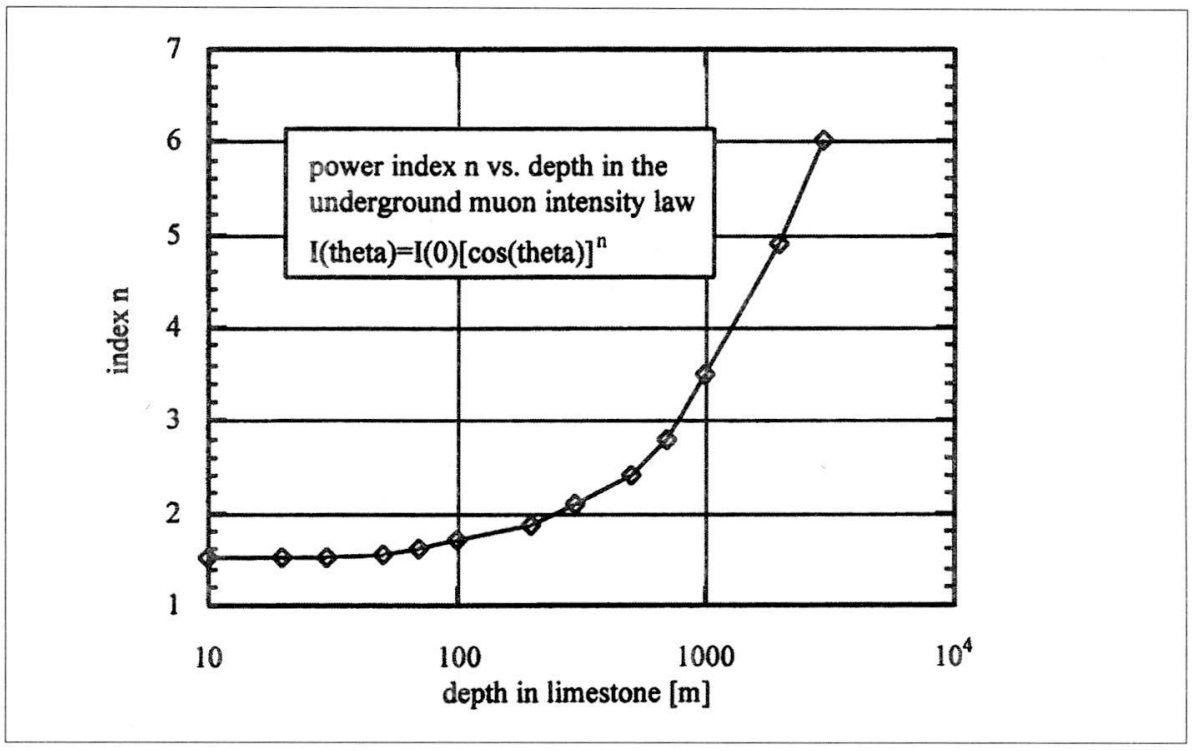

Fig. 6 - Power index " $n$ " versus depth 
With these assumptions we may now calculate the integrated muon flux on $\mathrm{S}$ from the "sky" in a cave, because only the directions around $\left(\theta_{0}, \varphi_{0}\right)$ contribute. The integration is very simple:

$$
F_{\mu}=\int_{k y} I_{\mu}(\theta, \varphi) S d \Omega \approx S I_{\mu}\left(x_{0}\right) \frac{2 \pi}{n\left(x_{0}\right)+1}
$$

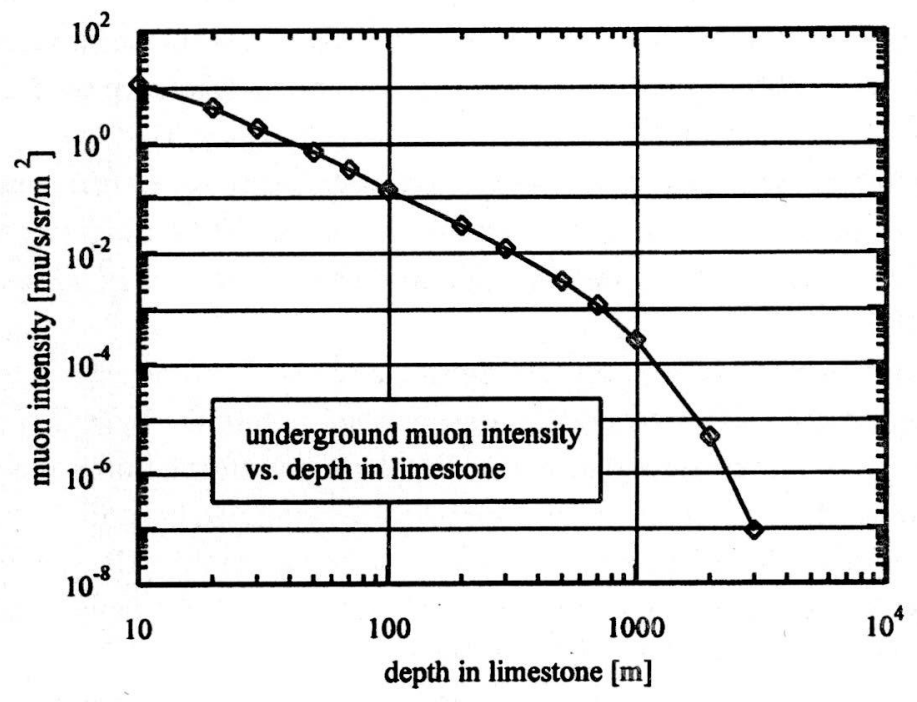

Fig. 7 - Muon intensity versus depth in limestone

Finally, we have our muon flux: this graphic Intensity-Depth, basic for our work, shows the number of muons as a function of "depth" (that is: the minimum rock thickness $\mathrm{x}_{0}$ ). We may now return to light.

\section{The lighting}

We are now going to calculate the average photons fluxes due to Cerenkov radiation, but we would like to put in a few words on what may be considered "lighting" from 
the point of view of the eye.

We have seen that the Cerenkov photons are disposed on a cone around the particle, which means that they reach a detector (or the cave floor) in very short $(<1$ ns) pulses. Their power (energy/time) may be very strong: for instance the outside Cerenkov radiation associated with Extensive Air Shower (mainly electrons Cerenkov radiation in the high atmosphere air) is larger than the average night light, which means that if we measure nocturnal light with high time resolution instruments, we see pulses from the sky overwhelming the light coming from the stars. The Cerenkov HE gamma ray observatories do exactly this (Aglietta et al., 1992).

But the physiological system cannot make this short pulses detection (they integrate the light fluxes on "long" time intervals, a tenth of a second), and looking at the night sky we cannot see the hundreds of strong flashes that, each second, touch our eyes.

Also caves lighting acts as a series of short flashes: if they were sufficiently near in time (as in the case of neon bulbs, that switch on and off 50 times per second) the light would appear us as a continuous illumination, but if they were rare we would see flashes.

The light reflection on walls poses other problems.

The reflected fraction of incoming photons, and not purely the light flux emitted by a source, is of extreme importance: actually the sight information is mainly due to the reflected light. For this reason, generally speaking, we have to estimate the reflected photons, which may strongly contribute to the lighting. If, say, the cave walls are perfect reflectors the photons are never absorbed: each new contribution is added and the cave light intensity diverges...

This question is easy to solve: let us call " $\mathrm{r}$ " the ratio between reflected and incident light on rock. If $\mathrm{I}_{0}$ is the incident light intensity the first reflection gives $r \times I_{0}$ : and so on. The total amount is:

$$
I=I_{0}+r \times I_{0}+r^{2} \times I_{0}+r^{3} \times I_{0}+\ldots=\frac{I_{0}}{1-r}
$$

Given that $0<\mathrm{r}<1$, the total illumination may increase appreciably, and it diverges if $r=1$ : but the rock reflectivity is generally very low and the reflected light appears to be negligible in our rude calculations.

\section{The light from air}

The first contribution to the caves illumination is the Cerenkov radiation in air. 
The muons must have at least $4 \mathrm{GeV}$ to start the emission and $15 \mathrm{GeV}$ to attain the plateau of production, 25 photons per meter. But the light production depends on the energy spectrum of muons, which depends on the depth! We shall not take this fact into account; the assumption that all the muons are well above the Cerenkov threshold is surely wrong but it is easy to show that the over-estimation of light flux it gives is negligible in our approach.

Each muon travels a length $\mathrm{R}_{C}(\theta, \varphi)$ from the cave wall to the detector surface $S$, and during the flight releases photons at a constant rate $\Gamma_{\mathrm{c}}$.

The total light flux on the horizontal surface $\mathrm{S}$ is then:

$$
L=\int_{k k y} S \cos \vartheta R_{C}(\theta, \varphi) \Gamma_{c} F_{\mu} d \Omega
$$

That obviously depends on the cave shape $R_{C}(\theta, \varphi)$ around $S$. Let us simplify again: we consider the surface $S$ oriented in the direction of $x_{0}$ to neglect the cosq contribution, and we assume " $\mathrm{D}$ " as the typical dimension of the cave in the direction of $\mathrm{x}_{0}$. For example, $\mathrm{D}$ will be the cavern height if we are below a plateau. In this case the light flux becomes:

$$
L=S D \Gamma_{c} F_{\mu}
$$

That is, a very simple and useful formula.

\section{Some examples}

We consider now some special cases. To make a comparison let us see this table, partially taken from (Lang, 1974).

Table 5 - A comparison of fluxes from natural or artificial light sources

\begin{tabular}{|l|c|c|c|}
\hline \multicolumn{1}{|c|}{ Source } & $\begin{array}{c}\text { Light flux } \\
{\left[\mathrm{W} \mathrm{m}^{-2}\right]}\end{array}$ & $\begin{array}{c}\text { Light flux } \\
{[\mathrm{lx}]}\end{array}$ & $\begin{array}{c}\text { Light flux } \\
{\left[\text { photons } \mathrm{m}^{-2} \mathrm{~s}^{-1}\right]}\end{array}$ \\
\hline Sun (at zenith) & $1.33 \times 10^{3}$ & $9 \times 10^{5}$ & $3 \times 10^{21}$ \\
\hline Night sky & $7 \times 10^{-7}$ & $5 \times 10^{-4}$ & $2 \times 10^{12}$ \\
\hline $6^{\mathrm{m}}$ star & $2.5 \times 10^{-10}$ & $1.7 \times 10^{-7}$ & $6 \times 10^{8}$ \\
\hline Candle at 10 km & $1.1 \times 10^{-12}$ & $7.5 \times 10^{-10}$ & $3 \times 10^{6}$ \\
\hline Candle on the Moon & $8 \times 10^{-22}$ & $5 \times 10^{-19}$ & $1.7 \times 10^{-3}$ \\
\hline
\end{tabular}

We have considered here some examples of light fluxes, averaging on $560 \mathrm{~nm}$ photons, which means a conversion factor given by 


$$
1 \mathrm{~lx}=3.4 \times 10^{15} \text { photons } \mathrm{m}^{-2} \mathrm{~s}^{-1}
$$

The light flux produced by a sixth-magnitude star is especially interesting because it is defined as the minimum energy flux from a point-like source detectable by human eyes. Considering a standard pupil ( $4 \mathrm{~mm}$ of diameter) this means that the human eye must receive around $10^{4}$ photons $\mathrm{s}^{-1}$ to obtain a visual sensation: but we are going to return to it at the end of this work.

We have used a "candle" with the same light power of the Candela SI unit.

Let us go to practical examples, considering at first the Grotta Gigante (hereafter "Type A cavern"). It is a very huge cavern near Trieste, with $D=100 \mathrm{~m}, \mathrm{x}_{0}=15 \mathrm{~m}$. These parameters in the graphic Intensity-Depth give us the typical muons flux inside the cave, $20 \mathrm{~m}^{-2} \mathrm{~s}^{-1}$ (but note that it is a coarse estimation, because a cave geometry like this does not fit with the approximations used to calculate the muon flux).

The light production of each muon crossing the cave is roughly 2000 photons: this means that the expected light flux is $4 \times 10^{4}$ photons $\mathrm{m}^{-2} \mathrm{~s}^{-1}$, or $1.6 \times 10^{-14}$ $\mathrm{Wm}^{-2}$.

The natural cosmic ray illumination of this Type A cave is then a 20 billionth of night sky light, or 0.1 thousandth of a $6^{\mathrm{m}}$ star light deposition...

We consider now a Type B cavern with parameters $200 \mathrm{~m}$ from the surface and $10 \mathrm{~m}$ of height $\mathrm{D}$ : we have a muon flux of $0.08 \mathrm{~m}^{-2} \mathrm{~s}^{-1}$ each with its 250 photons disposed as a "plate" (the cone is very flat because the Cerenkov angle is $1.4^{\circ}$ ) of $25 \mathrm{~cm}$ of radius around it. The average lighting of the cave is then 20 photons $\mathrm{m}^{-2} \mathrm{~s}^{-1}$.

In comparison with Grotta Gigante the reduction is by a factor 10 due to the height, and by a factor 200 due to muons flux.

If the cave cross-section is $100 \mathrm{~m}^{2}$ (that is it has a cubic or spherical shape) it is crossed by, say, ten muons per second: the light is released in "pulses" Poissonianly distributed with average time interval $0.1 \mathrm{~s}$.

At last we consider a Type $\mathrm{C}$ cavern, a small conduit $(\mathrm{D}=1 \mathrm{~m})$ at large distance from the surface $\left(\mathrm{x}_{0}=500 \mathrm{~m}\right.$ ): it has only 0.006 muons $\mathrm{m}^{-2} \mathrm{~s}^{-1}$ (a crossing muon each 3 minutes) and a natural average illumination of only 0.15 photons $\mathrm{m}^{-2} \mathrm{~s}^{-1}$. They are very low fluxes but note that, in any case, they are easy to detect (Badino et al., 1981). 


\section{The light from water}

Table 2 shows us that the minimum muon energy for light production is reduced to $50 \mathrm{MeV}$, and the light production increases to 20000 photons per meter.

For these two reasons the water contribution to the illumination in caves is so large that the main neutrino experiments now running use it (in artificial cavern or in deep sea and lake waters) for particles detection. The example of SuperKamiokande is astonishing: this Japanese neutrino detector (Fukuda et al., 1998) consists in a hall (17 m of radius and $40 \mathrm{~m}$ high) filled with 50 thousand tons of water, 1000 meters below the top of Mt. Ikenoyama!

We may again use the equation

$$
L=S \Gamma_{c} F_{\mu} R_{C}(\vartheta, \varphi)
$$

Considering $R_{C}(\theta, \varphi)$ no longer describing the cave structure but describing the water-filled part of a cave. As above, we define $\mathrm{D}$ has the dimension of the water basin along the $\mathrm{x}_{0}$ direction.

As a matter of fact we have a new problem: the muon energy loss in air was negligible, but its loss in water is not. A water-filled cave like Grotta Gigante (Type A) has a muon flux corresponding to $15 \mathrm{~m}$ of depth in rock in the upper part $\left(20 \mathrm{~m}^{-2} \mathrm{~s}^{-1}\right)$, but to $15 \mathrm{~m}$ of rock plus $100 \mathrm{~m}$ of water (roughly $40 \mathrm{~m}$ of rock equivalent) at the bottom: the flux is then reduced to $2 \mathrm{~m}^{-2} \mathrm{~s}^{-1}$. To estimate the total illumination we may assume an average muons flux of $10 \mathrm{~m}^{-2} \mathrm{~s}^{-1}$, each muon releasing two million photons during the crossing. The total average flux becomes $2 \times 10^{6}$ photons $\mathrm{m}^{-2} \mathrm{~s}^{-1}$, or $10^{-12} \mathrm{Wm}^{-2}$. This corresponds to the light of a candle $10 \mathrm{~km}$ away... But we are going to see that, in special conditions, this flux may give visual sensation.

Note that these photons are emitted on a very large cone $\left(41^{\circ}\right)$; this means that the lighting structure not only is stronger than in the case of air, but it has a very different structure and uniformity.

The case of illumination in conduits below the water table is also interesting.

We may consider the case of a Type $B$ cave, $D=10$ at $200 \mathrm{~m}$ of depth. The muon flux is $0.08 \mathrm{~m}^{-2} \mathrm{~s}^{-1}$, but each muon now produces $2 \times 10^{5}$ photons: the average illumination of this water storage is 2000 photons $\mathrm{m}^{-2} \mathrm{~s}^{-1}$.

We conclude considering Type $C$, the small conduit $(D=1 \mathrm{~m})$ at large distance from the surface $\left(x_{0}=500 \mathrm{~m}\right)$. With its small flux of 0.006 muons $\mathrm{m}^{-2} \mathrm{~s}^{-1}$ it has an average illumination of 15 photons $\mathrm{m}^{-2} \mathrm{~s}^{-1}$. It is obvious that in this case to speak of "average illumination" has small sense: the light is given in rare, strong photons pulses. 


\section{The light from rock}

Crystals (small or big) are other materials that can release light when crossed by charged particles. Let us see their contribution to caves lighting, speaking only about the most common type, the calcite.

The Cerenkov radiation in calcite is stronger than in water by a factor two, due to the higher refractive index (see Table 2), but the light release phenomenology main change is the light diffusion: the Cerenkov light in water is strongly organised into a cone around the muon, whereas in calcite it is diffused everywhere by the internal reflections on micro-crystal surfaces. This also causes a stronger absorption that depends, in a complex way, on micro-crystal dimensions and impurities.

Let us introduce a parameter of rock transparency, the average depth of photons absorption, i.e. roughly the depth $\lambda$ at which the light may arrive. It may range from micron in rock to many centimetres in pure micro-crystal deposits.

It is easy to show that the outgoing light from an absorbing medium is almost exactly the same produced in the depth from 0 to $\lambda$. In the case of cosmic rays underground this means that the light coming from the walls is estimated by:

$$
L=S \lambda \Gamma_{c} F_{\mu}
$$

The value may vary of orders of magnitude and giving examples as above makes no sense. What we may say is that micro-crystal deposits behave more efficiently than water in caves illumination, but only up to a depth of $\lambda$, and that generally speaking we have to add the contribution of water veils on walls. They have a typical depth of 50$100 \mu \mathrm{m}$ (Badino, 1995) and release 1-2 photons when crossed by a muon.

\section{The radioactive beta decays}

The last chapter has prepared the introduction to the next light source in caves: the Cerenkov light emission in crystals by electrons produced in beta decays. The limestone rocks contain traces of radioactive nuclei, mainly Uranium-238, Thorium-232, and Potassium- 40 .

Let us study at first the ${ }^{40} \mathrm{~K}$ contribution: a nucleus that mainly performs a beta decay to the stable nuclide ${ }^{40} \mathrm{Ca}$. The beta decay consists in the transformation of a neutron into a proton plus an electron, that is emitted with a maximum energy $\mathrm{E}_{0}$, which has a specific value for each nucleus. The electrons have energy spectra that in the upper part are described by:

$$
P(E) d E \approx k E^{2}\left(E_{0}-E\right)^{2} d E
$$


Table 6 shows the physical parameters for ${ }^{40} \mathrm{~K}$ and the corresponding symbol that we are going to use from now on.

Tab. 6 - Physical parameters for ${ }^{40} \mathrm{~K}$

\begin{tabular}{|l|c|}
\hline \multicolumn{1}{|c|}{${ }^{40} \mathrm{~K}$} & Properties \\
\hline Mode of decay & Beta (89\%), EC (11\%) \\
\hline E decay $[\mathrm{MeV}]$ & 1.311 \\
\hline Natural abundance $\mathrm{r}_{\mathrm{K} 40}[\%]$ & 0.0117 \\
\hline Average life for beta decay $\tau_{\mathrm{K}}[\mathrm{y}]$ & $1.41 \times 10^{9}$ \\
\hline Atomic mass $\mathrm{M}_{\mathrm{K}}[\mathrm{kg}]$ & $6 \times 10^{-26}$ \\
\hline
\end{tabular}

From the graphic, showing the energy spectrum of electrons emitted during the ${ }^{40} \mathrm{~K}$ beta decay, we may see that the main part of electrons are emitted with kinetic energy equal to half of the total energy beta decay, in this case $0.5 \mathrm{MeV}$. The other line shows the mean Cerenkov light emission for each electron energy range in calcite, weighted with electrons ranges, energy spectra, Cerenkov radiation phenomenology: obviously the spectrum is shifted to right in comparison with the previous one because the radiation output increases with the particle energy.

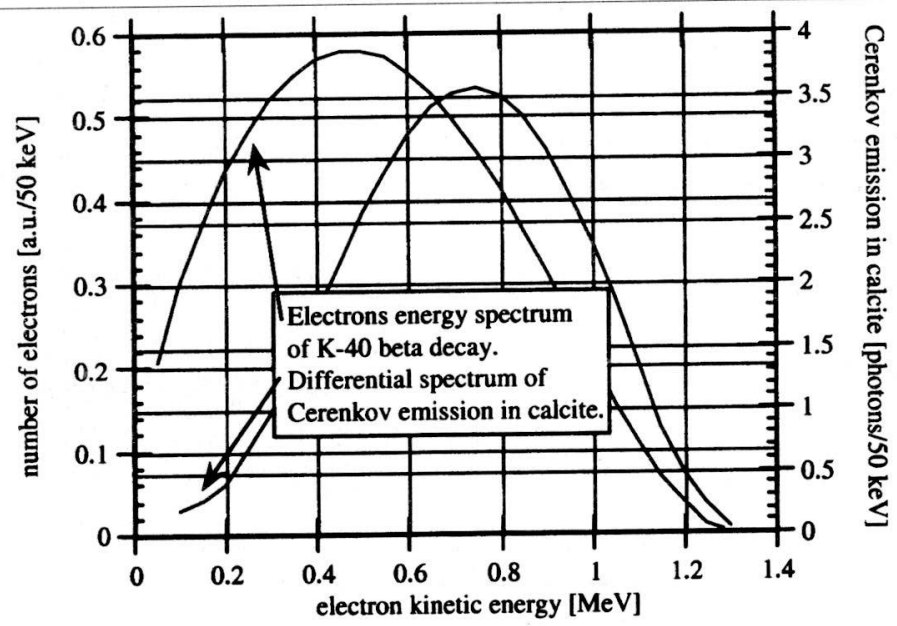

Fig. 8 - The energy spectrum of electrons emitted by ${ }^{40} \mathrm{~K}$ beta decay, and the corresponding light emission in calcite.

The emitted electron crosses the matter, but it interacts strongly, ionising other atoms and losing energy. Table 7, adapted from (Jelley, 1959), gives the average penetration $\lambda_{\mathrm{el}}$ in calcite as a function of electron total energy: 
Tab. 7 - Average penetration $\lambda_{\text {el }}$ in calcite as a function of electron total energy:

\begin{tabular}{|c|c|}
\hline $\begin{array}{c}\text { Electron kinetic energy } \mathrm{E}_{\mathrm{k}} \\
{[\mathrm{MeV}]}\end{array}$ & $\begin{array}{c}\text { Range } \lambda_{\mathrm{el}} \\
{[\mathrm{mm}]}\end{array}$ \\
\hline 0.2 & 0.15 \\
\hline 0.4 & 0.46 \\
\hline 0.6 & 0.81 \\
\hline 0.8 & 1.19 \\
\hline 1 & 1.58 \\
\hline 1.2 & 1.98 \\
\hline
\end{tabular}

It is possible to see that the electron paths are very short, around a millimetre of length. We may estimate that only the atoms decaying in the first rock layer (some millimetre thick) may contribute to lighting, and also that the total light production (In Table 2: 30 photons $\mathrm{mm}^{-1}$ ) is limited to some tenth of photons per decay. Let us calculate it better.

\section{The light from beta rays}

During its flight the electron emits Cerenkov light, but it has a kinetic energy that approaches the energy threshold for light emission. So, to calculate the average total light emitted per decay (we are going to call " $\varepsilon_{\text {light }}$ " this parameter), we have to take into account:

- the electrons spectrum,

- the electron energy loss in flight,

- the length of the electron walk,

- the energy dependence of Cerenkov emission.

A numerical approach that considers all these phenomena gives us the results resumed in Table 8.

Table 8 - Average photon production in underground materials due electron from ${ }^{40} \mathrm{~K}$ beta decay.

\begin{tabular}{|l|c|}
\hline \multicolumn{1}{|c|}{ Medium } & $\begin{array}{c}\varepsilon_{\text {light }} \\
\text { [photons decay }\end{array}{ }^{-1}$ ] \\
\hline Water & 17 \\
\hline Calcite $(\mathrm{n}=1.68)$ & 23 \\
\hline Calcite $(\mathrm{n}=1.49)$ & 15 \\
\hline
\end{tabular}

The light production is then around twenty photons per decay in calcite and in water: the production increase due to the higher refractive index of crystal is almost compensated by the shorter electron range to due to the higher density.

Let us calculate the frequency of decays. The potassium contents " $\mathrm{q}_{\mathrm{K}}$ " in limestone 
is widely variable: we are going to consider the example of Gran Sasso, the rock of which is well studied due to the presence of underground neutrino laboratories. Ambrosio et al. (1995) report a content of $0.1 \%$, that is, each kilogram of limestone contains one gram of potassium, or $1.17 \times 10^{-7} \mathrm{~kg}$ of ${ }^{40} \mathrm{~K}$, that corresponds to $1.5 \times 10^{18}$ atoms, each one with atomic mass $M_{K}=6 \times 10^{-26} \mathrm{~kg}$. The number of decay (activity) per kilogram of rock is:

$$
A_{K}=\frac{q_{K} r_{K 40}}{M_{K}} \frac{1}{\tau_{K}}
$$

With the average life " $\tau_{\mathrm{K}}$ " given in Table 6 we may estimate that in a kilogram of Gran Sasso limestone there are 40 beta decays each second: the density of activity $A_{K}$ is then $40 \mathrm{~Bq} / \mathrm{kg}$.

Let us calculate the photon production rate per mass unit, that we will call " $\Gamma_{\text {light }}$ ".

We have that:

$$
\Gamma_{\text {light }}=\frac{q_{K} r_{K 40}}{M_{K}} \frac{1}{\tau_{K}} \times \varepsilon_{\text {light }}=A_{K} \varepsilon_{\text {light }}
$$

The previous calculations and estimations give us a production rate in rock of

$$
\Gamma_{\text {light }}=70 \text { photons } \mathrm{kg}^{-1} \mathrm{~s}^{-1}
$$

coming from the electrons emitted in ${ }^{40} \mathrm{~K}$ beta decay.

\section{The light from potassium EC-gammas}

Another way that permits light release from ${ }^{40} \mathrm{~K}$ decay is the interaction of its gamma rays in rock. Let us describe the process.

As we have seen in Table 6 the ${ }^{40} \mathrm{~K}$ performs mainly beta decay, that is, it emits an electron with a very short range in rock. But sometimes the ${ }^{40} \mathrm{~K}$ decays from an electron capture (EC): no electron is then emitted but the energy is released in a monochromatic gamma ray with quite high energy, $1.46 \mathrm{MeV}$.

The probability of this process is

$$
\varepsilon_{E C}=0.105
$$

A gamma photon of this energy is able to cross a much larger rock thickness than the electron does. It interacts with matter by photoelectric effect, Compton scattering of electrons or creating electron-positron pairs. The general process is quite complex and has to be studied by Montecarlo simulations, but we may give some analytical estima- 
tion, using averaged data and the fact that the Compton effect in rock dominates in the energy range 0.1-1 MeV (Segre, 1965).

When an electron-positron pair is created almost no light is released: the average electron energy in not sufficient to give Cerenkov effects, the positron is almost immediately annihilated and gives other gamma rays that have low probability to give light photons, due to their low energy and isotropic emission.

The Cerenkov emission comes from the electrons scattered by gammas. They have an almost flat energy spectrum, that is, they may have any energy between zero and 1.46 $\mathrm{MeV}$, i.e. the average kinetic energy of these Compton electrons is around $0.7 \mathrm{MeV}$; after the scattering the photon continues its flight with an energy lowered by the energy transfer to the electron and may scatter other electrons. So, if in the first interaction the energy transfer to the electron is low, the gamma may perform other Compton interactions. To take very roughly into account this multiple production we may consider that the average energy of a scattered electron is $2 / 3$ of the maximum, that is, $1 \mathrm{MeV}$. The gamma attenuation length $\lambda_{\mathrm{g}}$ is $0.2 \mathrm{~m}$ at $1.4 \mathrm{MeV}$ (Particle Data Group, 1986), that is, an $\mathrm{EC}{ }^{40} \mathrm{~K}$ decay within some tenths of centimetre inside the rock releases a gamma that may be able to exit the walls.

Note the difference from the beta-decay: in the former case only the most superficial decays can participate to the light process production; now, due to the long gamma ray range, much deeper parts of rock are involved.

We may give a rude estimation of the gamma flux from rock. As before, let us call $\mathrm{A}_{\mathrm{K}}$ the density of activity of ${ }^{40} \mathrm{~K}$ in the rock. The number of decays per second per kilogram of rock (that is the gamma production rate) in the EC capture channel is then $\varepsilon_{\mathrm{EC}} \mathrm{A}_{\mathrm{K}}$.

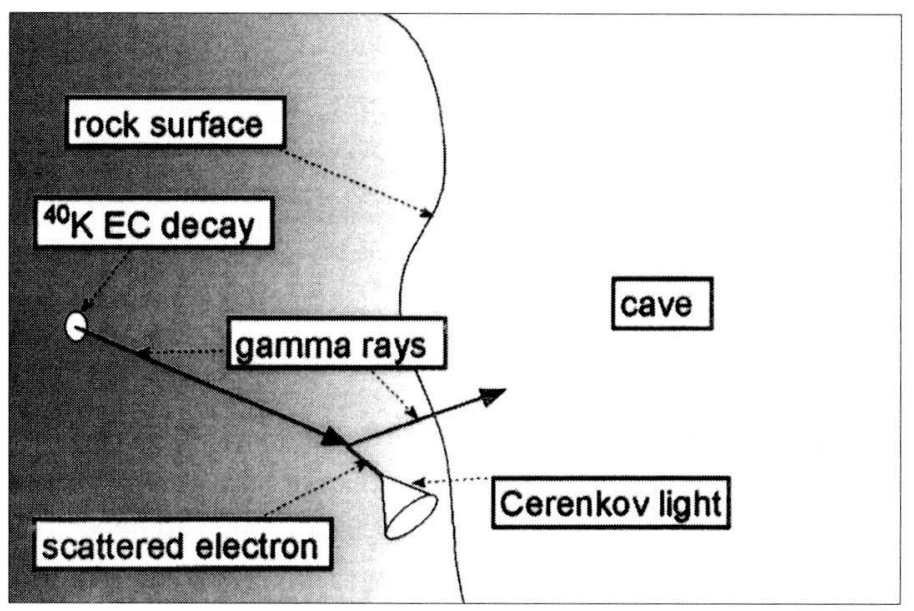

Fig. 9 - General process of light production from ${ }^{40} \mathrm{~K}$ $E C$ : if the emitted gamma scatters an electron just below the rock surface some photons may escape. 
From Table 7 we have that the Compton electrons they may produce in rock (average energy $1 \mathrm{MeV}$ ) have a range $\lambda_{\mathrm{el}}$ of $1.5 \mathrm{~mm}$.

To calculate the light released by the electron it is necessary to take into account the energy loss in flight, the energy dependency of Cerenkov emission and the energy spectrum. Numerical calculation gives us that a $1 \mathrm{MeV}$ electron in calcite emits 30 Cerenkov photons along its $1.5 \mathrm{~mm}$ range.

We have assumed a density of activity $\mathrm{A}_{\mathrm{K}}$ of $40 \mathrm{~Bq} / \mathrm{kg}$ in the beta channel. In the EC channel it is then

$$
A_{E C}=\varepsilon_{E C} A_{K} \approx 4 \quad \mathrm{EC}_{\text {gammas kg }}{ }^{-1}
$$

If we assume that each gamma is able to produce, on average, one electron (that is, 30 photons) along its path, we may estimate the light release per kilogram of rock

$$
\Gamma_{\text {light,EC }}=120 \text { photons } \mathrm{kg}^{-1} \mathrm{~s}^{-1}
$$

But it is now necessary to estimate how many of these photons are able to escape from rock.

\section{The EC photons escape from rock}

Let us consider a surface $S$ up to a depth $\lambda_{\mathrm{g}}$. The gamma rate production inside it is then

$$
\Gamma_{g, p r o d}=\varepsilon_{E C} A_{K}\left(\rho_{r} S \lambda_{g}\right)
$$

Only half of these gammas are emitted in the direction of the surface and may really go out. It would be necessary to calculate the probability of gamma absorption for each depth inside the wall and integrate it, and in the next chapter we will solve a similar problem obtaining a correction factor of 0.25 . We may then estimate that the number of gammas flowing out from our surface $S$ each second is

$$
\Gamma_{g, f l u x}=0.25 \varepsilon_{E C} A_{K}\left(\rho_{r} S \lambda_{g}\right)
$$

The rock is not transparent and the electron range is very short so neither electrons nor the Cerenkov photons they produce can exit the wall, unless the gamma-electron interaction takes place in the first transparent rock "skin" with optical depth $\lambda$. We may estimate that the probability that the gamma (with photon attenuation length 
$\lambda_{\mathrm{g}}$ ) interacts in the last $\lambda$ meters of rock is

$$
P_{\text {compon }} \approx \frac{\lambda}{\lambda_{g}}
$$

This slightly overestimates the probability because in principle the formula is true only for gammas crossing the surface perpendicularly. Neither the Compton electron produced in this way is, in general, perpendicular to the surface and consequently the Cerenkov light may be emitted in "wrong" directions; we may use an additional factor $1 / 2$ to take these effects into account. Finally, we may estimate that the Compton electrons produced each second in $\mathrm{S}$ and able to release light are

$$
\left.\Gamma_{\text {compron }} \approx \frac{1}{2} 0.25 \varepsilon_{E C} A_{K}\left(\rho_{r} S \lambda_{g}\right) \frac{\lambda}{\lambda_{g}} \text { [electrons s }{ }^{-1}\right]
$$

We have seen that the average electron emits 30 photons. We have to distinguish between two cases, that is whether $\lambda$ (rock transparency) is larger than $\lambda_{\mathrm{el}}$ (electron range) or not. In the former case each electron will release the maximum number of photons that will flow out from the wall. That is:

$$
\begin{aligned}
& \left.\Gamma_{\text {light }}=\frac{1}{2} 0.25 \varepsilon_{E C} A_{K}\left(\rho_{r} S \lambda_{g}\right) \frac{\lambda}{\lambda_{g}} \times 30 \text { [photons }^{-1}\right] \\
& \text { for } \lambda>\lambda_{e l} \approx 0.0015 \mathrm{~m}
\end{aligned}
$$

But if the electron range is longer than $\lambda$ the Cerenkov photons emitted deeper than $\lambda$ are absorbed and only the last part of emission is able to flow out the rock:

$$
\begin{aligned}
& \Gamma_{\text {light }}=\frac{1}{2} 0.25 \varepsilon_{E C} A_{K}\left(\rho_{r} S \lambda_{g}\right) \frac{\lambda}{\lambda_{g}} \times 30 \frac{\lambda}{\lambda_{e l}}\left[\text { photons s}{ }^{-1}\right. \text { ] } \\
& \text { for } \lambda<\lambda_{e l} \approx 0.0015 \mathrm{~m}
\end{aligned}
$$

That is, the maximum release decreases proportionally to $\lambda$ if $\lambda>\lambda$ el $=1.5 \mathrm{~mm}$ and proportionally to $\lambda^{2}$ if $\lambda<1.5 \mathrm{~mm}$.

For example, let us calculate the Cerenkov release with $\lambda=0.001 \mathrm{~m}, \mathrm{~A}_{\mathrm{K}}=40 \mathrm{~Bq}$ $\mathrm{kg}^{-1}$ (Gran Sasso limestone). We have:

$$
\frac{\Gamma_{\text {light }}}{S}=\frac{1}{2} 0.25 \times 0.1 \times 40 \times(2700 \times 0.2) \frac{0.001}{0.2} \times 30 \times \frac{0.001}{0.0015} \approx 30 \text { photons } \mathrm{m}^{-2} \mathrm{~s}^{-1}
$$

If $\lambda=0.1 \mathrm{~mm}$ the light flux will be reduced by a factor 100 , that is only 0.3 photons will flow each second from a square meter of wall.

Compton scattering in the cave air, instead, cannot produce light because the electron energy is in any case much smaller than the energy threshold given in Table 2. 


\section{The lighting role of heavy nuclei decays}

We have seen how beta and gamma rays may release small light production in rock. Let us see now the contribution of other important radioactive impurities in rock, the heavy nuclei ${ }^{238} \mathrm{U},{ }^{235} \mathrm{U}$ and ${ }^{232} \mathrm{Th}$.

These elements are unstable, long life nuclei present in traces almost everywhere: typical abundances (widely variable) of Uranium and Thorium are $10^{-6}$ in effusive rocks and $10^{-7}$ in organisms and limestone: we are going to consider the last figure for our estimations.

Due to its shorter half-life ${ }^{235} \mathrm{U}$ abundance is only $0.72 \%$ of ${ }^{238} \mathrm{U}$.

These three nuclei decay almost exclusively by Alpha decay, emitting an Alpha particle (an Helium nucleus) of $4 \mathrm{MeV}$ and, very seldom $(<1 \%)$, some low energy gamma ray. In these conditions no light release is possible, unless by the luminescence channel (see below).

But the first alpha decay is only the beginning of a series of decays, because the products are not stable. Each one of the three nuclei is then the chief of a family of unstable nuclei that decay in various manners up to produce Lead nuclei.

For example, let us see in Table 9 the main radioactive family, the ${ }^{238} \mathbf{U}$ decay chain.

Table 9 - The ${ }^{238} \mathrm{U}$ decay chain.

\begin{tabular}{|c|c|c|c|c|}
\hline $\begin{array}{c}{ }^{238} \mathrm{U} \\
\text { decay family }\end{array}$ & Mean life $\tau$ & Half life $\mathrm{T}_{1 / 2}$ & Main mode of decay & $\begin{array}{c}\text { Mean charged particle } \\
\text { energy (e or } \alpha)\end{array}$ \\
\hline${ }^{238} \mathrm{U}$ & $6.5 \times 10^{9} \mathrm{y}$ & $4.5 \times 10^{9} \mathrm{y}$ & alpha & $4.2 \mathrm{MeV}$ \\
\hline${ }^{234} \mathrm{Th}$ & & 24 days & beta & $50 \mathrm{keV}$ \\
\hline${ }^{234} \mathrm{~Pa}$ & & $1.17 \mathrm{~min}$. & beta & $820 \mathrm{keV}$ \\
\hline${ }^{234} \mathrm{U}$ & & $2.5 \times 10^{5} \mathrm{y}$ & alpha & $4.9 \mathrm{MeV}$ \\
\hline${ }^{230} \mathrm{Th}$ & & $7.5 \times 10^{4} \mathrm{y}$ & alpha & $4.7 \mathrm{MeV}$ \\
\hline${ }^{226} \mathrm{Ra}$ & & $1600 \mathrm{y}$ & alpha & $4.9 \mathrm{MeV}$ \\
\hline${ }^{222} \mathrm{Rn}$ & & 3.8 days & alpha & $5.6 \mathrm{MeV}$ \\
\hline${ }^{218} \mathrm{Po}$ & & 3.1 min. & alpha & $6.1 \mathrm{MeV}$ \\
\hline${ }^{214} \mathrm{~Pb}$ & & $27 \mathrm{~min}$. & beta & $336 \mathrm{keV}$ \\
\hline${ }^{214} \mathrm{Bi}$ & & $20 \mathrm{~min}$. & beta & $1270 \mathrm{keV}$ \\
\hline${ }^{214} \mathrm{Po}$ & & $164 \mu \mathrm{s}$ & alpha & $7.6 \mathrm{MeV}$ \\
\hline${ }^{210} \mathrm{~Pb}$ & & $22 \mathrm{y}$ & beta & $17 \mathrm{keV}$ \\
\hline${ }^{210} \mathrm{Bi}$ & & 5 days & beta & $390 \mathrm{keV}$ \\
\hline${ }^{210} \mathrm{Po}$ & & 138 days & alpha & $5.3 \mathrm{MeV}$ \\
\hline
\end{tabular}

The general process that gives Lead from Uranium decay takes hundreds of thousand years, due to the high half-life of ${ }^{234} \mathrm{U}$, but in a rock every decay stage is present. At the equilibrium the activity of each isotope has to be exactly the same of the progenitor, so we may consider that, on average, each Uranium or Thorium decay releas- 
es all the decay products of its decay chain.

We have seen that the alpha decay does not contribute to light production: in Table 10 we see the physical parameters of heavy nuclei decays that are able to create a light signal.

Table 10 - Physical parameters of heavy nuclei decays able to create a light signal.

\begin{tabular}{|c|c|c|c|}
\hline Decay Chains & $\begin{array}{c}\text { Nuclide } \\
\text { (decay energy, } \mathrm{MeV} \text { ) }\end{array}$ & $\begin{array}{c}\varepsilon_{\text {light }} \\
\text { [photons/decay] }\end{array}$ & $\begin{array}{c}\text { Gamma energy [MeV] } \\
\text { (gamma/decay) }\end{array}$ \\
\hline \multirow[t]{6}{*}{${ }^{238} \mathrm{U}$ family $\rightarrow{ }^{206} \mathrm{~Pb}$} & ${ }^{234} \mathrm{Th}(0.27)$ & 0 & $0.1(10 \%)$ \\
\hline & ${ }^{234} \mathrm{~Pa} \mathrm{(2.20)}$ & 39 & $\begin{array}{l}0-1 \mathrm{MeV}(0.1 \%) \\
1-2 \mathrm{MeV}(0.2 \%)\end{array}$ \\
\hline & ${ }^{214} \mathrm{~Pb}(1.0)$ & 12 & 0 \\
\hline & ${ }^{214} \mathrm{Bi}(3.27)$ & $\begin{array}{l}60 \text { (+5 from } \\
\text { gammas) }\end{array}$ & $\begin{array}{c}0.6(46 \%) \\
1.1(15 \%) \\
1.2-1.4(10 \%) \\
2.2(5 \%)\end{array}$ \\
\hline & ${ }^{210} \mathrm{~Pb}(0.06)$ & 0 & 0 \\
\hline & ${ }^{210} \mathrm{Bi}(1.16)$ & 15 & 0 \\
\hline \multirow[t]{4}{*}{${ }^{235} \mathrm{U}$ family $\rightarrow{ }^{207} \mathrm{~Pb}$} & ${ }^{231} \mathrm{Th}(0.39)$ & 0 & 0 \\
\hline & ${ }^{227} \mathrm{Ac}(0.04)$ & 0 & 0 \\
\hline & ${ }^{211} \mathrm{~Pb}(1.37)$ & 21 & $0.8(4 \%)$ \\
\hline & ${ }^{207} \mathrm{Tl}(1.42)$ & 21 & 0 \\
\hline \multirow[t]{4}{*}{${ }^{232} \mathrm{Th}$ family $\rightarrow{ }^{208} \mathrm{~Pb}$} & ${ }^{228} \mathrm{Ra}(0.05)$ & 0 & 0 \\
\hline & ${ }^{228} \mathrm{Ac}(2.13)$ & 38 & 0 \\
\hline & ${ }^{212} \mathrm{~Pb}(0.57)$ & 4 & $0.3(50 \%)$ \\
\hline & ${ }^{212} \mathrm{Bi}(2.25)(64 \%)$ & 40 & 0 \\
\hline
\end{tabular}

The second column gives the decaying isotopes and the decay energy, the fourth the energy of gammas released during the beta decays with the probability of emission. It is possible to see that the role of these gammas is negligible.

The third column shows the average photon release per decay due to the electron Cerenkov emission. We have numerically included the main physical effects, as we did in Table 8 to estimate the light flux from ${ }^{40} \mathrm{~K}$ beta decay (emission spectra, ranges, Cerenkov release).

The light releases are in general very small except those of ${ }^{228} \mathrm{Ac},{ }^{234} \mathrm{Pd},{ }^{212} \mathrm{Bi}$ and mainly ${ }^{214} \mathrm{Bi}$ due to the energy of emitted electrons. The role of ${ }^{214} \mathrm{Bi}$ is also increased by the presence of energetic gamma rays that may release light $(\approx 5$ photons per decay) as we have seen in ${ }^{40} \mathrm{~K}$ EC.

In conclusion, we may estimate that each ${ }^{238} \mathrm{U},{ }^{235} \mathrm{U}$ and ${ }^{232} \mathrm{Th}$ decay is able to give respectively 300,50 and 100 photons.

Let us estimate the number of decays per rock kilogram for these three nuclei. We hav 


$$
\begin{aligned}
& A_{U 238}=\frac{q_{U 238}}{M_{U 238}} \frac{1}{\tau_{U 238}}=\frac{10^{-7}}{4 \times 10^{-25}} \frac{1}{2.0 \times 10^{17}}=1.2 \text { decay kg-1 } \mathrm{s}^{-1} \\
& A_{U 235}=\frac{q_{U 235}}{M_{U 235}} \frac{1}{\tau_{U 235}}=\frac{0.0072 \times 10^{-7}}{4 \times 10^{-25}} \frac{1}{3.2 \times 10^{16}}=0.06 \text { decay kg } \mathrm{kg}^{-1} \\
& A_{T h 232}=\frac{q_{\text {Th232 }}}{M_{\text {Th232 }}} \frac{1}{\tau_{\text {Th232 }}}=\frac{10^{-7}}{4 \times 10^{-25}} \frac{1}{6.4 \times 10^{17}}=0.4 \text { decay kg-1 } \mathrm{s}^{-1}
\end{aligned}
$$

With these data (rock with Potassium abundance of $1000 \mathrm{ppm}$, Uranium and Thorium of $0.1 \mathrm{ppm}$ ) we may estimate the total photon release in rock, given in Table 11 .

Table 11 - Total photon release in rock

\begin{tabular}{|c|c|}
\hline Nucleus & $\begin{array}{c}\Gamma_{\text {light }} \\
\text { [photons s} \\
\end{array} \mathrm{kg}^{-1}$ ] \\
\hline${ }^{40} \mathrm{~K}$ beta & 700 \\
\hline${ }^{4} \mathrm{~K} \mathrm{EC}$ & 120 \\
\hline${ }^{238} \mathrm{U}$ & 150 \\
\hline${ }^{235} \mathrm{U}$ & 2,5 \\
\hline${ }^{232} \mathrm{Th}$ & 33 \\
\hline
\end{tabular}

We have to treat now the process of photons exit from rock.

\section{The beta decay photons escape from rock}

Let us consider an element of calcite at depth " $\mathrm{r}$ " from the surface. We suppose that calcite produces light isotropically; this light is subsequently absorbed with average free walk $\lambda$ that may be considered the depth of the rock transparent "skin".

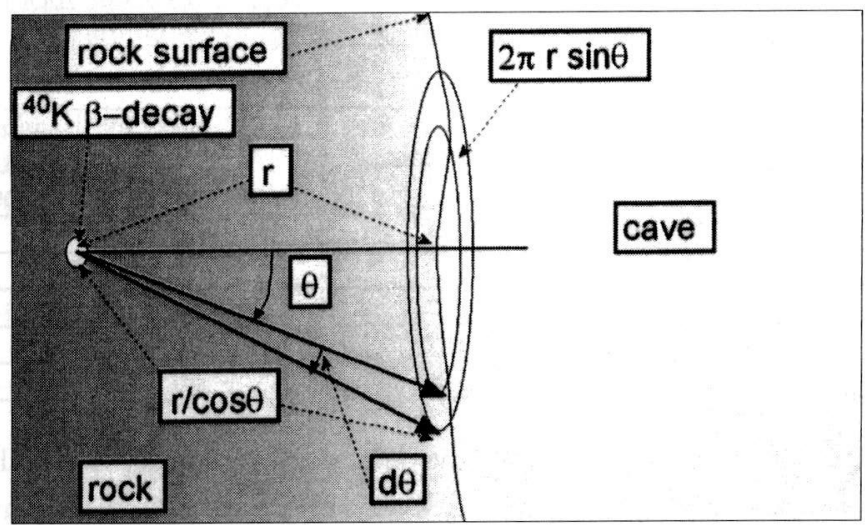

Fig. 10 - The physical parameters involved in the calculation of light emission from the ${ }^{40} \mathrm{~K}$ beta decay inside the rock. 
The total flux exiting rock is given by the not-trivial integral:

$$
\begin{aligned}
& F_{p h}(r) d r=\frac{1}{4 \pi r^{2}}\left(\Gamma_{\text {light }} \rho_{r} S d r\right) \int_{0}^{\pi} 2 \pi r^{2} \sin \theta \exp \left(-\frac{r}{\cos \vartheta} \frac{1}{\lambda}\right) d \theta= \\
& =\frac{1}{2}\left[\exp \left(-\frac{r}{\lambda}\right)-\frac{r}{\lambda} E i\left(\frac{r}{\lambda}\right)\right]\left(\Gamma_{\text {light }} \rho_{r} S d r\right)
\end{aligned}
$$

Where $\mathrm{Ei}$ is the exponential-integral function and the factor $1 / 2$ takes into account the fact that only half of the emitted photons fly in the direction of the surface and may escape. The flux is then reduced very quickly due to the rock absorption.

If we integrate this function from $\mathrm{r}=0$ (the wall surface) up to infinity we obtain the total light out-coming from rock:

$$
\int_{0}^{\infty} F_{p h}(r) \approx 0.26\left(\Gamma_{\text {light }} \rho_{r} S \lambda\right)
$$

That is, the emission from rock corresponds to $1 / 4$ of the total light produced up to the depth $\lambda$. With the usual assumption ( $\lambda$ rock transparency parameter, $\varepsilon_{\text {light }}$ number of photons per decay, A number of decays per kilogram of rock) we may write that the total outgoing light flux is then

$$
\frac{F}{S}=0.26 \rho_{r} \lambda \Gamma_{\text {light }}=700 \lambda \varepsilon_{\text {light }} A
$$

Let us suppose that we have walls with $\lambda=0.1,1,10 \mathrm{~mm}$ : than the light flux from rock is given by Table 12, calculated with the assumption that Potassium abundance in

\begin{tabular}{|c|c|c|c|c|}
\hline Nucleus & 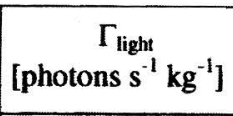 & $\begin{array}{c}\text { Light Flux for } \\
\lambda=0.1 \mathrm{~mm} \\
\text { [photons } \mathrm{m}^{-2} \mathrm{~s}^{-1} \text { ] }\end{array}$ & $\begin{array}{c}\text { Light Flux for } \\
\lambda=1 \mathrm{~mm} \\
\text { [photons } \mathrm{m}^{-2} \mathrm{~s}^{-1} \text { ] }\end{array}$ & $\begin{array}{c}\text { Light Flux for } \\
\lambda=10 \mathrm{~mm}^{-2} \\
\text { [photons } \mathrm{m}^{-1} \text { ] }\end{array}$ \\
\hline${ }^{40} \mathrm{~K}$ beta & 700 & 50 & 500 & 5000 \\
\hline${ }^{40} \mathrm{KEC}$ & 120 & 0.3 & 30 & 450 \\
\hline${ }^{238} \mathrm{U}$ chain & 150 & 10 & 100 & 1000 \\
\hline${ }^{235} \mathrm{U}$ chain & 3 & 0.2 & 2 & 20 \\
\hline${ }^{232} \mathrm{Th}$ chain & 40 & 3 & 28 & 280 \\
\hline
\end{tabular}
rock is $1000 \mathrm{ppm}$, and that of Uranium and Thorium is $0.1 \mathrm{ppm}$.

Table 12 - A summary of light production from radioactive families inside rock with typical abundance, as a function of rock transparency.

The light flux is linearly proportional to the radio nuclide abundances (scaling it to different abundances is trivial) and generally to $\lambda$, with the exception of gamma processes (for example the ${ }^{40} \mathrm{~K}$ EC) that depends on $\lambda$ if $\lambda>\lambda_{\mathrm{el}}=1.5 \mathrm{~mm}$ and $\lambda^{2}$ if $\lambda<1.5 \mathrm{~mm}$. 


\section{Radioactive decays in water}

The Potassium and Uranium contents in fresh water are widely variable: the heavy nucleus abundance ( $0.03 \mathrm{ppm}$ in sea water) ranges from 0.024 to $200 \mathrm{ppb}$ (Kathren, 1984). We may assume as "standard" values respectively $0.001 \mathrm{ppm}$ for Uranium and $1 \mathrm{ppm}$ for Potassium respectively.

This means that we are now assuming that for both radio-nuclides the water contents are 100 and 1000 times smaller than we assumed for rock $(0.1 \mathrm{ppm}$ and $0.1 \%$ respectively). Also the activities must therefore scale by the same factors; if we include all the detailed processes performed by electrons (or, for EC, by gamma quantum) in water ( $\mathrm{n}=1.33$, electron ranges more than two times longer) we may adapt Tables 8 and 10 to obtain the light released by each decay process.

Table 13 - Light released by each decay

\begin{tabular}{|c|c|c|}
\hline Decay Chains & $\begin{array}{c}\text { Nuclide } \\
\text { (decay energy, MeV) }\end{array}$ & $\begin{array}{c}\varepsilon_{\text {light }} \\
\text { [photons/decay] }\end{array}$ \\
\hline${ }^{238} \mathrm{U}$ family $\rightarrow{ }^{206} \mathrm{~Pb}$ & ${ }^{234} \mathrm{~Pa}(2.20)$ & 50 \\
\hline & ${ }^{214} \mathrm{~Pb}(1.0)$ & 8 \\
\hline & ${ }^{214} \mathrm{Bi}(3.27)$ & 90 \\
\hline & ${ }^{214} \mathrm{~Pb}(1.0)$ & 11 \\
\hline & & 20 \\
\hline${ }^{235} \mathrm{U}$ family $\rightarrow{ }^{207} \mathrm{~Pb}$ & ${ }^{211} \mathrm{~Pb}(1.37)$ & 21 \\
\hline & ${ }^{207} \mathrm{Tl}(1.42)$ & 48 \\
\hline & & 52 \\
\hline${ }^{232} \mathrm{Th}$ family $\rightarrow{ }^{208} \mathrm{~Pb}$ & ${ }^{228} \mathrm{Ac}(2.13)$ & 0 \\
\hline & ${ }^{212} \mathrm{Bi}(2.25)(64 \%)$ & 17 \\
\hline & ${ }^{212} \mathrm{~Pb}(0.57)$ & 35 \\
\hline${ }^{40} \mathrm{~K}$ EC gamma $\rightarrow$ compton & $(\sim 1.5$ total $)$ & \\
\hline
\end{tabular}

If we include the activity of the "standard" water taken into consideration, we may calculate (Table 14) the light released in water.

Table 14 - Light released in water

\begin{tabular}{|c|c|c|}
\hline Type of decay & $\mathrm{A}\left[\mathrm{Bq} \mathrm{kg}{ }^{-1}\right]$ & $\begin{array}{c}\Gamma_{\text {light }} \\
{\left[\text { photons } \mathrm{kg}^{-1} \mathrm{~s}^{-1}\right]}\end{array}$ \\
\hline${ }^{40} \mathrm{~K}$ beta & 0.4 & 7 \\
\hline${ }^{60} \mathrm{~K}$ EC & 0.04 & 1.4 \\
\hline${ }^{238} \mathrm{U}$ & 0.012 & 2 \\
\hline
\end{tabular}


The Potassium contribution dominates, the main Uranium isotope is strong and the Thorium and different Uranium isotopes are almost zero.

It seems a small light release, but water transparency, generally very high, can transform this small effect into a strong light source in large underground water basins: a water like that, filling a Type $B$ cave $(D=10 \mathrm{~m}$, see above) releases more than 1 million photons per second, some 2000 photons $\mathrm{m}^{-2} \mathrm{~s}^{-1}$ on walls, roughly the same flux than the Cerenkov light.

Obviously, the behaviour (coherence, directionality, rise time) of the two light emissions are completely different, but it is so easy to detect that it is a source of random noise in Neutrino experiments that have to use highly purified water o reduce the energy threshold of data acquisition.

\section{Neutron light sources}

Others more exotic sources of light are surely working in caves.

The ${ }^{238} \mathrm{U}$ undergoes self-fission decay, producing neutron fluxes and a complex of gamma ray with $7 \mathrm{MeV}$ of total energy. The neutron flux is very small (3.2 per fission) but it represents an important background source in neutrino astronomy because the neutron capture on $\mathrm{H}$ releases a gamma pulse of $2.2 \mathrm{MeV}$, and capture on other nuclei $(\mathrm{C}, \mathrm{O}, \mathrm{Ca}, \mathrm{N})$ releases gamma ray pulses with energies ranging around 5-10 MeV (Aglietta et al., 1986).

These high-energy gamma rays may create high-energy electrons by pair production or Compton scattering that can produce Cerenkov light if in water or crystals.

In underground laboratories carved in granite rocks the neutron fluxes are typically 0.5 neutrons $\mathrm{m}^{-2} \mathrm{~s}^{-1}$ (Aglietta et al., 1986), but in sedimentary rocks they are at least one order of magnitude smaller. In Gran Sasso, for example (Bellotti et al. 1985), the flux is 0.03 neutrons $\mathrm{m}^{-2} \mathrm{~s}^{-1}$.

We may estimate that the light production has an order of magnitude of one photon per hour per square meter, really difficult to detect !

\section{A "secondary" effect: the luminescence}

We have almost finished our outlook on "standard" cave illumination, that is the light fluxes produced in any type of cave. Other processes may be occasionally at work: the most important one is probably luminescence (Forti \& Hill, 1986).

Many materials are able to emit light when relatively cool, for instance electronically excited gases in neon lamps, inorganic crystals ("phosphors") in kinescopes, fluorescent lamps and fireflies. The basic mechanism is simple: input energy is absorbed by an isolated "centre" (atom, ion or molecule) that becomes excited and then emits some photons when it returns to the unexcited state. Many types of luminescence are 
possible, depending upon the excitation mechanisms (fracture, X-rays, UV, heating and so on) and on the time delay between excitation an emission: if the time delay is perceptible the substance is called "phosphorescent".

These processes are not so usual and efficient, but many minerals act as phosphors: barite, fluorite, willemite, scheelite and so on.

The most common are generally sulphide-type, consisting in a matrix of micro-crystal sulphides with some impurities that act as activators in a very complex way. One of the main candidates to participate to the illumination processes in caves is surely the gypsum (calcium sulphide), very widely associated with the limestone caves speleogenesis. Anyway, also calcite and aragonite appear to have fluorescent and phosphorescent behaviour in particular cases (Cigna, 1962; Forti \& Hill,1986).

The atoms excitation underground may come from: UV photons from Cerenkov radiation, energetic electrons produced by ionisation by the passage of a muon in the material, alpha, $\mathrm{X}$ and gamma rays from heavy nuclei decays.

The first term is surely active because many calcites appear to be UV-fluorescent, and we have seen above that the main part of Cerenkov emission is in the first UV region. These minerals act as wavelength shifters, transforming an invisible UV photon into one or two visible photons. The total effect is then small, increasing the Cerenkov total release in rock by a factor 2-3.

But in presence of luminescent minerals the ionisation is surely the most important excitation process. The passage of a single charged particle through matter creates thousands of free electrons per centimetre of flight. If the material has ionisationluminescent properties it may release a light pulse by far stronger than the Cerenkov one. Then the terms depending on the radioactive decays (Potassium, Uranium and Thorium) may be very large due to the large energy releases involved, that may be in part transferred to light production: we must remember that the global transformation of ${ }^{238} \mathrm{U}$ into ${ }^{206} \mathrm{~Pb}$ releases $60 \mathrm{MeV}$, that corresponds to the energy of tens of millions of light photons!

In real cases, for instance, the liquid and plastic scintillators, widely used for particle detection, are based on these processes and consist essentially of an organic substance doped with an activator (PPO) that releases UV photons on free electrons capture and a wavelength shifter (POPOP) that absorbs these photons and re-emits others as blue light. These scintillators, strongly optimised for light emission, have a luminescence light release a factor 100 higher than the Cerenkov contribution (Particle Data Group, 1986)!

Obviously we do not know which are the cave minerals able to release light if excited by a crossing particle (it is a very simple study to carry out) although it seems reasonable to suppose that all luminescent minerals are sensitive to ionisation. We may 
also suppose that they are not optimised for light emission: we may probably estimate that the light released by luminescent minerals is one order of magnitude larger than that released by the Cerenkov processes.

In any case we are speaking of light coming from the "skin" of the walls; we have already seen that this type of lighting, generally speaking, gives a very small contribution due to low rock transparency.

On the other hand the most efficient light producer in caves, the almost pure karst water, does not produce any luminescent effects. We may therefore estimate that the light coming from luminescence processes can be appreciable only in very peculiar cases.

\section{The illumination of under-ice Antarctica lakes}

In recent year very huge, under-ice lakes have been discovered under the central Antarctica ice sheet. The main basin is the Lake Vostok, roughly $3700 \mathrm{~m}$ below the surface, $200 \mathrm{~km}$ long, covering an area of $14,000 \mathrm{~km}^{2}$ and in some places at least 500 $\mathrm{m}$ deep. The water temperature have to be around $-2.5^{\circ} \mathrm{C}$. Around 70 other subglacial water-bodies are known to exist, that probably form a vast hydrological system, that may be a unique habitat for ancient bacterial life (Kapitsa et al., 1996).

At this depth there is no residual light flux from the surface because the typical photons average walk inside ice ranges from few to some tens of meters: but the muons can reach those waters.

From our graphic Intensity-Depth (Fig. 7) we may estimate the residual muon flux in $10^{-8}$ muons $\mathrm{m}^{-2} \mathrm{~s}^{-1}$; the typical length to cross the lake is around $500 \mathrm{~m}$, but we have to include also the Cerenkov contribution of crossed ice just above the lake. Probably a total light release of $2 \times 10^{7}$ photons per muon is not far from reality. The total light flux in those lakes is some $0.1-0.2$ photons $\mathrm{m}^{-2} \mathrm{~s}^{-1}$, which appears to be unusable for life.

We do not know anything about origin and chemistry of those waters, so no other light flux (from beta decay, fluorescence and so on) can be estimated here.

\section{Lights at giant depths}

We may conclude our overview on caves darkness looking for light presence in "caves" at enormous depth. It is reasonable to think that only in the first $50-80 \mathrm{~km}$ of depth some cavities may exist, filled with water or extremely highly pressurised gases: below this level, the rock plasticity probably suppresses "caves" formation. In these cavities the processes described just above (light production from beta decay 
and neutron captures) are surely active with their typical fluxes.

The cosmic ray muons cannot attain those depth, but neutrinos can easily (they cross the Earth without interaction), even though they cannot give any light signal being electrically neutral. However sometimes a neutrino interacts with rock producing a muon, which travels for some length interacting with rock and, in case, with caves. At giant depth we can therefore detect a constant flux of muons produced isotropically in the hundreds of meters of rock surrounding the observation point (Noell,1971). This flux, that allows us to study the high-energy neutrinos (and allows the HE Neutrino Astronomy), is about $2 \times 10^{-7}$ muons $\mathrm{m}^{-2} \mathrm{~s}^{-1}$.

A water bubble of 1 ton (Type D cavern) is then crossed by 5 muons per year, say, it "sees" one thousand photons per year, that is $10^{-20} \mathrm{lux}, 1 / 50$ of the light given on Earth by a candle on the Moon. It is really dark.

This is the residual external light, a rare flash that sometimes overwhelms the lighting of local beta decays...

\section{Physiological effects}

The process of vision is very complex and poorly understood, but fortunately its features are outside the purposes of this paper. But we want to give some overlook to understand, finally, why the caves appear so dark to us.

Every "eye" consists essentially of a light collector, a photosensitive receiver that transforms the image into pulses and a system that reconstructs the image converting it in a "sensation". The first part is essentially represented by the pupil, whose dimensions widely change between vertebrates depending upon the typical working light fluxes: owls have pupils very large... The second part is the retina, an extremely complex nervous system (it is said "a second brain") whose photosensitive receptors are cells (rod and cone) that transform light produced molecular changes into nervous excitation. The last part is the brain, that creates an image sensation based on nervous data: almost nothing is known about this last phase.

Our question is: what is the smallest photons number that will produce a visual response in complete darkness? Experiments have shown that a single rod cell responds to the absorption of single photon acting on a single molecule. However this response does not produce visual sensation: the brain would be submerged by data. To have a visual sensation it is necessary to have a minimal response at about the same time (but the retina time resolution is low) in at least five rod cells within a small area of the retina. (D'Arcy \& Porter, 1962)

This shows that the "vision" is something different from the simple photon detection, and in fact it has completely different purposes. It is not strange that caves appear to us as dark: the general features of caves illumination do not fit with the vision para- 
meters selected outside by life evolution.

We have seen two features of the caves lighting: the average flux is very low and is released in extremely short pulses. At first we have to note that the fluxes are, almost in many cases, not so small to be undetectable by physiological systems, and that the pulsed behaviour of detecting systems is not so strange for life purposes: let us remember the bats ultrasonic detector. But physiological systems appear to have a low time resolution response and they "smoothes" light fluxes on "large" times, losing the main way to detect these pulses on account of their extremely short time duration.

It is interesting to discuss the visibility of Cerenkov pulses into the eye. Some pioneering attempt was made forty years ago by D'Arcy and Porter (D'Arcy \& Porter, 1962) to study the visual detection of single relativistic cosmic ray muons. Many other studies in this field were then carried out after astronauts of Apollo program reported unexpected visual sensations (light flashes) (McNulty, 1971; Charman \& Rowlands,1971). The exact mechanism has been a subject of controversy, because it is not easy to discriminate between Cerenkov radiation and direct ionisation and excitation of molecules at the retina. In these years an experiment has been devoted to these studies in Mir (SilEye) and another (ALTEA) is planned in International Space Station (Avdeev et al., 2001).

Let us return underground, as in the Grotta Gigante. The average muon flux (20 $\left.\mathrm{m}^{-2} \mathrm{~s}^{-1}\right)$ and the eye's surface $\left(5 \mathrm{~cm}^{2}\right)$ allow us to estimate in $100 \mathrm{~s}$ the average time between two muons interactions in an eye. This means that, roughly one time per minute, we are going to see a production of 50,000 photons in our eyes, directly against retina. If we compare it with the fluxes from a $6^{\mathrm{m}}$ star we may see that we have roughly the same intensity: it is difficult, but we may see it! So, it is probable that a part of phosphenes (the luminous impression due to excitation of the retina by other than impingement of light) that we may see in caves darkness is the visible part of cave illumination...

\section{Shooting photos in natural underground light}

We have seen that the light fluxes underground are easy to detect for modern instruments. It is very natural to ask if it is possible to make photos...

Let us calculate the exposure times to obtain pictures in natural underground light. Let us call " $k$ " the lens aperture (f/k) and T the exposure time in seconds. It is widely used the concept of Exposure Value, a number referring to the combination of $\mathrm{k}$ and T, which gives the same resulting exposure on the film. It is defined for $100 \mathrm{ASA}$ film as 


$$
E V=\log _{2}\left(\frac{k^{2}}{T}\right)=3.32 \log \left(\frac{k^{2}}{T}\right)
$$

The EV is correlated with the light flux F (in lux) by

$$
F=2.6 \times 2^{E V}
$$

This gives us a relation between the light flux and the shooting parameters on a 100 ASA film:

$$
T=2.6 \frac{k^{2}}{F}
$$

The flux $\mathrm{F}$ is the flux of light reflected from surface, not that impinging on it. Let us call " $\mathrm{r}$ " the surface albedo as above. In this case

$$
T[\text { years }]=3 \times 10^{8} \frac{k^{2}}{r F\left[\text { photons } \mathrm{m}^{-2} \mathrm{~s}^{-1}\right]}
$$

Table 15 - Light flux in various caves and exposure time to make a good picture

\begin{tabular}{|c|c|c|}
\hline Case & $\begin{array}{c}\text { Light flux F } \\
\text { [photons } \mathrm{m}^{-2} \mathrm{~s}^{-1} \text { ] }\end{array}$ & $\begin{array}{c}\text { Exposure time on a } \\
\text { 400 ASA film, } \mathrm{f} / 1.4 \\
\text { [years] }\end{array}$ \\
\hline Grotta Gigante (A, air) & $4 \times 10^{4}$ & $2 \times 10^{4}$ \\
\hline Type B (air) & 20 & $4 \times 10^{7}$ \\
\hline Type C (air) & 0.15 & $5 \times 10^{9}$ \\
\hline Type A (water) & $2 \times 10^{6}$ & 400 \\
\hline Type B (water) & 2000 & $4 \times 10^{5}$ \\
\hline Type C (water) & 15 & $5 \times 10^{7}$ \\
\hline Type D (water) & $3 \times 10^{-5}$ & $2 \times 10^{13}$ \\
\hline Lake Vostok & 0.2 & $4 \times 10^{9}$ \\
\hline
\end{tabular}

This playful table gives us the exposure time, in years, to make a good picture in the various cave types described above, with natural light on a 400 ASA film with lens aperture $\mathrm{f} / 1.4$, and $\mathrm{r}=0.2$.

We have considered here only the light flux coming from muons Cerenkov release in water or air, but not the contributions of rock as the Cerenkov light from muons in rock and in water veil on surface, or from beta decays, fluorescence effects and so on. 


\section{Conclusions}

The cosmic radiation and other secondary effects are able to release visible light in underground cavities, mainly by the Cerenkov effect. The illumination increases linearly with the cavity dimensions.

These light emissions are largely used for cosmic rays studies, especially in Underground Neutrino Observatories, and generally speaking are able to give physiological effects, but the need of noise reduction forces the brain to disregard such weak signals in the construction of a visual sensation. This is the reason why caves appear to us so dark.

It is easy, for us, to design "eyes" able to reconstruct images from light fluxes in caves, but they would be large and energetically expensive devices that could work only in large cavities. The Nature, more cleverly, seems to have chosen different and simpler ways to give good detectors of the surrounding world to the underground life.

\section{References}

AGLIETTA M. et al.,, 1986. - Low-energy Neutral-particle Detection in the Mont Blanc LSD Experiment. Nuovo Cimento 9C: 271-279.

AGLIETTA M. et al., 1992 - EAS-TOP: Results of Gamma-ray Astronomy at $10^{14} \mathrm{eV}$. Nuovo Cim. , 15C: 357-364.

AGLIETTA M. et al., 1995 - Neutrino-induced Single-muon Flux Measured by LVD at Gran Sasso. Proc. XXIV ICRC, Vol. 1-HE: 734-737.

AMBROSIO M. et al., 1995 - Vertical Muon Intensity Measured with MACRO at the Gran Sasso Laboratories. Phys. Rev. D 52: 3793-3802.

AVDEEV S. et al, 2001 - The SilEye Nuclei Cosmic Eay and Eye Light Flash Experiment on board the Mir Space Station. Proc. ICRC XXVII: 1745, 2001, 1745-1748.

BADINO G., 1995 - Fisica del Clima Sotterraneo. Mem. Ist. Italiano di Speleologia. 7, II: 41

BADINO G., GALEOTTI P., PERIALE L., SAAVEDRA O., 1981 - Time Delay between Parallel Muons. ICRC XVII, 10, 350-353. 
BELLOTTI E., BURASCHI M., FIORINI E. \& LIGUORI, C. 1985 - INFN Internal Report, Milano,

CECCHINI S., 2001 - Underground Experiments for Cosmic Ray Physics: Results and Future Projects. Nucl. Phys. B, Proc. Suppl. 97: 48

CIGNA A., 1962 - Sulla luminescenza di alcune stalattiti calcaree. Actes 2 Congr. Int. Spel., Bari-Lecce-Salerno 1958,1: 430/436.

CHARMAN W. \& ROWLANDS C. M., 1971 - Visual Sensation Produced by Single Fast Particle. Nature, 230: 574-576.

D'ARCY F. J. \& PORTER N. A., 1962 - Detection of Cosmic Ray $\mu$-Mesons by the Human Eye. Nature, 196: 1013-1014.

FORTI P.\& HILL C., 1986 - Cave Minerals of the World. NSS: 166

FUKUDA Y. et al., 1998 - Measurements of a Small Atmospheric $n_{m} / n_{e}$ Ratio. Phys. Lett. B, 433: 9-18.

GAISSER T., 1990 - Cosmic Rays and Particle Physics. Cambridge University Press: 79

HAYAKAWA S., 1969 - Cosmic Ray Physics. Wiley-Interscience: 398

JELLEY J., 1959 - Cerenkov Radiation. Pergamon Press: 21

KAPITSA A.P. et al., 1996 - A Large Deep Freshwater Lake beneath the Ice of Central East Antarctica. Nature 381: 684-686.

KATHREN R.L., 1984 - Radioactivity in the environment: sources, distribution and surveillance. Harwood Acad. Publishers.

LANG K., 1974 - Astrophysical Formulae. Springer-Verlag, , 572

NOELL W., 1971 - Vision. Enc. Brit. , 23, , 62

MCNULTY P., 1971 - Light Flashes Produced in the Human Eye by Extremely Relativistic Muons. Nature, 234: 110-112.

PARTICLE DATA GROUP, 1986 - Review of Particle Properties. Phys. Lett. 170B: 43

RICHTMYER F. \& KENNARD E., 1955- Introduction to Modern Physics. McGraw-Hill: 118 
SEGRE E., 1965 - Nuclei and Particles. Benjamin Inc.: 36

THEWLIS J., 1962 - Luminous Efficiency of Radiation. Enc. Dict. Of Phys., Macmillan Company, 4: 383

Received 15 September 2001

Accepted 31 December 2001 